\title{
ESTIMAÇÃO E PREDIGÃ̃o NOS MODELOS MISTOS NÃO BALANCEADOS
}

\author{
LUIS ALBERTO LOPEZ PEREZ
}

Estatí stico

Orientador: PROF. DR. ANTONIO FRANCISCO I EMMA

Tese apresentada a Escola superior de
Agricultura "Luiz de Queiroz", da
Universidade de são Paulo, para
obtençáo do litulo de Doutor em
Agronomia, Area de Concentração:
Estatistica e Experimentacão Agro-
nômica.

P I R A C I C A B A

Estado de São Paulo - Brasil

Dezembro - 1992 
Ficha catalográfica preparada pela Seção de Livros da Divisão de Biblioteca e Documentação - PCAP/USP

Lopez Perez, Luiz Alberto

L864e Estimaçăo e prediçăo nos modelos mistos năo balanceados. Piracicaba, 1992.

85p.

Tese - ESALQ

Bibliografia

1. Análise de variância - Componente 2. Estatística aplicada 3. Método da máxima verossimilhança 4. Modelo matemático I. Escola Superior de Agricultura Luiz de Queiróz, Piracicaba

CDD 519.535 


\section{ESTTMACÃO E PREDIGÃO NOS MODELOS MISTOS NÃO BALANCEADOS}

Aprovado en $02-12-1992$

Comissão Julgadora:

- Prof. Dr. Antonio Francisco Ienma.............. ESALA/USP

- Prof. Ir. Lécio Barbin.................... ESALA/USP

- Profo. Dra. Meria Cristina Stolf Nogueira........ ESALa/USE

- Prof. In. Lucio Benedicto Kroll................. UNESP

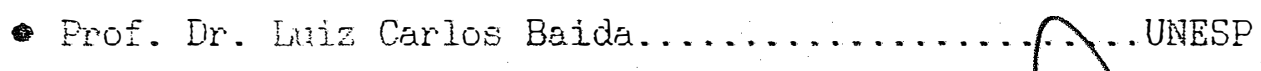

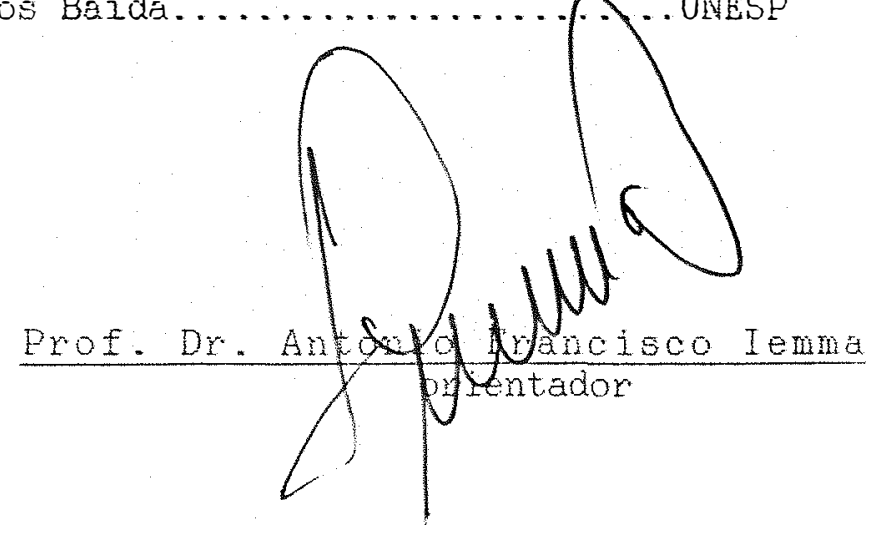


DEDICATÓRIA

- Ao meu Pai, na sua memória.

LUIS ALFREDO LOPEZ

- À minha nãe

BENEDICTA PEREZ

- a clara, david alberto e daniel F.

- Aos meus Irmãos. 


\section{AGRADECIMENTOS}

- Ao Prof, Dr. Antonio Frandisoo Iema, pela sua orientacão e apoio en todos os sentidos cono professor e amigo.

- Aos meus professores do Departamento de Matemética e Estatística, em particular à Dra. Clarice G. B. Demetrio, Dr. Décio Barbin e Dr. Humberto de Campos.

- Aos colegas do prograna de Doutorado.

- A mestre Maria Izalina Ferreira Alves.

- Aos Anigos Jairo Jimenez, Gladys de Jimenez e Ficicardo Montalban.

- Aos meus sobrinhos Denis e René. 
RESUMO

SUMMARY

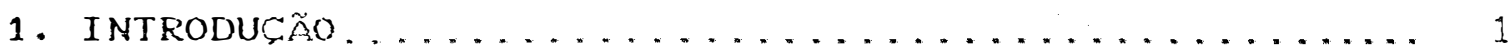

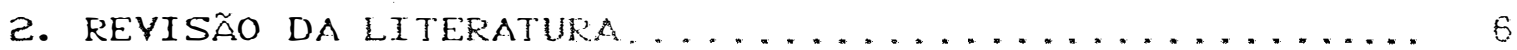

2.1. Idéias Gerais......................

2. 2. Resumo Hitorico...................... 14

3. RESULTADOS PRELIMIMARES ....................... 17 ?

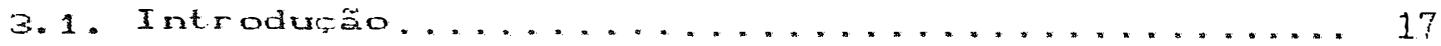

3.2. Modelo Estatistico..................... 17

3.2.1. Valores Esperados e matrizes de variâncias e covariâncias........... 18

3. 3. Estimaçáa dos Ereitos Fixos e Aleatórios...... 19

3.4. Caracterizacäo dos Preditores Lineares........ 24

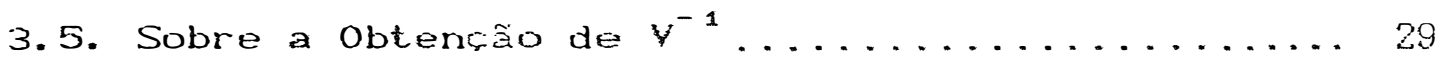

3.6. Formas Explicitas do ElLUE e do BLUP nos

Modelos com Dois Critérios de Classificacão.... 35

4. ESTIMAC̃̃O DOS COMFONENTES DE YARIÂNCIA.......... 40

4.1. Introdusão ....................... 40

4. 2. Método da Máxima Verossimilhança............ 40

4. 3. Cálculo Assintćtico dos Componentes de

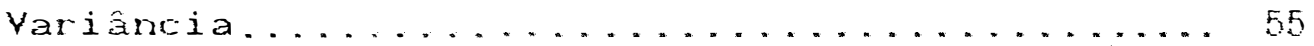

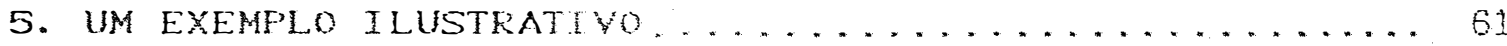

6. CONCLUSOES.............................. 7 ]

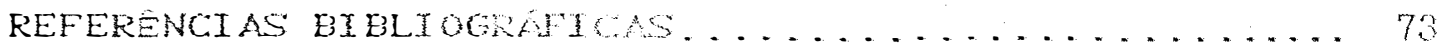

APENDICE FROGRAPI $W$ MATRIX IML 


\section{ESTIMACAO E PREDIGAO NOS MODELOS \\ MISTOS NAO BALANCEADOS}

Autor: LUIS ALBERTO LOPEZ PEREZ

Orientador: PROF. DR. ANTONIO FRANCISCO IEMMA

\section{RESUMO}

Este trabalho apresenta un estudo sobre as funcôes e preditores lineares nos modelos de efeitos mistos, com estrutura de dados balanceados e desbalanceados.

Propĩe-se um método alternativo para a estimacão dos preditores lineares, baseado nos multiplicadores de Lagrange os quais dependem linearmente da estimacão dos efeitos fixos e aleatórios do modelo. A estimasão desses efeitos dependem da estrutura da matriz associada de variâncias e covariâncias, por isto apresenta-se una metodologia visando simplificar sua construão, assin como o cálculo de sua inversa.

Para a estimarão dos compnentes da variânoia, o modelo é escrito em terwos das decomposióses ortogonais lineares, através das quais se estabelece uma relacão entre os sub-indices associados aos efeitos do modelo e os produtos de Kronecker das natriass identidades de orden $=(a s)$ e, quadradas de elenentos unitarios de orden $s\left(J_{5}\right)$, com a qual óloulo dos estimadores de méxime verossimi lhan a dos compontes de varînoia associados fica Ensivelmente simpificado. 


\title{
ESTIMATION AND PREDIOTION WITH MIXED UNBALANCED MODELS
}

\author{
Author: LUIS ALEERTO LOFEZ PEREZ \\ Adviser: PROF. DR. ANTONTO FRANCISCO IEMMA
}

\section{SUMMARY}

This work makes a study about the linear functions and predictors in models with mixed effects with balanced and unbalanced datums.

Here is presented an alternative method for the estination of the linear predictors based on the Lagrange's multiplicators, which depend linearly on the estimation of the fixed and aleatory effects of the model. The estimation of these effects depend on the structure of the associate variances and covariances matrix; that's why, a methodology is presented to facilitate its construction and the calculation of its inverse.

For the estimation of the variance's components, the model is written in terms of linear orthogonal decompsitions, that allow to stablish a relationship between the subindex associated with the effects of the model an the konecher products of the identities matrices of order $s$ ( $I$ ) and the square matrices of order $s\left(J_{s}\right)$, notation this, that smlties the caloulation of the maximum likelihood estimators of the cosociate veriane"s components. 


\section{INTRODUG}

A teoria estatí stica desenvolvia para a análise de modelos lineares com estrutura de dados desbalanceada, têm sido amplamente estudada e difundida nas últimas décadas, enfatizando preferencialmente os modelos de efeitos fixos como podem ser vistos em livros relativamente novos como os de DODGE (1985), HOCKING (1985), SEARLE (1987), dentre outros.

Dcorre porém, em muitas situacöes experimentais, que un ou mais fatores podem estar associados a processos de amostragem, já que não é possí vel levar em consideracăo toda a popularăo. Fatores desse tipo são ditos aleatórios, e caracterizam os modelos de efeitos aleatórios.

Pode acontecer ainda que sejam estudados de forma simultânea, efeitos fixós e aleatórios, caracterizando-se assim os modelos mistos. Situacões como essas säo muito frequentes em experimentos com seres vivos, tanto ná área animal como vegetal.

Segundo SCHEFEÉ (1959), esse tipo de modelo foi anplamente estudado por Fisher em 1918, gue denominou modelo de componentes de variancia, com grande repercusero nos estudos da genética quantitativa. A partir dai, forem dindidos en muitas outras disciplinas do conheoimento oientífico, mas considerando apenas os arranjos experinentais om estrutura de dados 
balanceados. Apenas a partir dos estudos de HENDERSON (1953), foram aboráados os modelos de componentes de variància com estruturas de dados desbalanceados.

Motivados pelas múltiplas aplicacões, mitos pesquisadores contribuiram no desenvolvimento de novas técnicas e procedimentos para estimar os componentes de variância nos modelos mistos, sobressaindo-se os trabalhos de HARTLEY \& FAO (1967) que apresentan o clássico nétodo da máxima verossimilhancáa.

Outros trabalhos clássicos são os de PATTERSON \& THOMPSON (1971) que desenvolveram o método da máxima verossimilhanca restrita (REML) e o procedimento desenvolvido por RAO (1971-1972) e LA MOTTE (1973) conhecido como MINQUE, dentre outros.

Nos modelos mistos três aspectos são fundamentais:

i) Estimasão dos efeitos fixos.

ii) Estimacão dos efeitos aleatórios.

iii) Estimacão dos componentes de variância.

Nesta sequência, no presente trabalho, pretende-se abordar o modelo dos efeitos mistos com estrutura balanceada e não balanceada, desenvolvendo-se os resultados teóricos básicos para satisfazer tais aspectos, de acordo com a seguinte sequência: No capítulo 2 apresenta-se uma revisão da literatura na qual verificou que nấo há muitos trabalhos desenvolvidos referente aos aspectos (i) e (ii), e que, no entanto, a maioria dos trabalhadores abordam os modelos mistos visando encontrar metodologis para estimara dos componentes de variencia ou seja (10 agecto ini) 
No capítulo 3, caracteriza-se a forma geral de modelo de efeitos mistos, introduzindo a distribuicão normal nos resí duos, embora muitos dos nétodos de estimarão das componentes não exijan essa propriedade distribucional.

A seguir, nas estimativas pelo nétodo dos mínimos quadrados generalizados, são obtidas as estruturas gerais dos melhores estimadores lineares não viesados (BLUE- best linear unbiased estimator), e dos melhores preditores lineares -(BLUP best linear unbiased preditor); neste trabalho apresenta-se un Forna altemativa para a obten za dos preditores.

O estudo dos preditores lineares näo tem sido aimde muto difundo embora tenha grandes aplicacoes práticas, principanente en melhoramento animal, por exemplo em programas de inseminaca artificial, quendo ae quer estudar os meritos genéticos dos reprodutores.

O preditor, por si só ú uariável aleatoria que 6 fumà de outro conjunto de variáveis aleatórias (geraimente os componentes de variância). Este fato pode dificultar o desenvolvimento teórico. No entanto, sob a suposicão de normalidade, o preditor pode ser entendido como uma esperanca condicional da distribuição nornal multivariada.

No entanto, no caso do BLUE, se faz notar neste trabsino, que quando a estrutura dos dados é balanceada e a matria associada aos efeitos fixos é de posto completo (caso dos modelos de nódias ou quando se faz reparametrizasăo), a estimativa dos parametros associada aos efeitos fixos, não vai depender dó natureza da matriz de componentes de variância. 
Neste capí tulo também são estudadas formas gerais para obtencão da inversa da matriz de componentes de variância, gerando-se uma "regra simples" no caso de dados balánceados e de modelos hierárquicos com estruturas desbalanceadas, simplificando sobremareira os cálculos computacionais.

Una vez caracterizada a inversa da matriz, finalmente no capí tulo 3, arresentam-se formas explí citas para se obter o BLUE e o BuUp nos modelos com un e dois oritérios de classificara. e aindá no caso de arranjos en blocos ao acaso, con estrutura de mécias. Com isto, procura-se dar ao leitor resultados imediatos nestes nodelos. Alén disso obsenva-se gue, se a estrutura dos dados for balanceada, e se a matriz associada aos efeitos fixos ś le posto coluna completo, entäo o BLUE nâa depende dos componentes aleatónios.

No capí tulo 4 são estudados métodos de estimarão dos componentes de variáncia, baseados na teoria normal e no nétodo da máxima verossimilhanca. Inicialmente são apresentadas "Kegras Práticas" para se construir matrizes ortogonais, que associadas às cominacões lineares dus componentes de variância, facilitám a estimativa de tais componentes, levando-se em consideracão a inveriânoia dos estimadores de máxima verossimilhansa. Observa-se, entã o, que este trabalio apresenta una contribuicão também neste sentido.

De forma genśrica, e através dos exemplos, são estimadas funcöes dos respectivos componentes, levando-se em conta a facilidade de estinaca dos componentes com base nas "Regras Práticas". 
Ademais säo apresentados os algori tmos de Newton Raphson e os Escores de Fisher para se estimar os componentes de variancia no caso de grandes amostras, ou aindàn no caso de näo ser possível escrever combinac̃es Iineares de componentes de viriancia da forma descrita anteriomente.

Finalmente leva-se a cabo un desenvolvimento computacional, usando o procedimentos MATRIX do SAS. 


\section{REVISÃO DE LITERATURA}

\subsection{Idéias gerais}

Nesta secão, são apresentados os trấlhos de um conjunto de pesquisadores que têm contribuí do no desenvolvimento

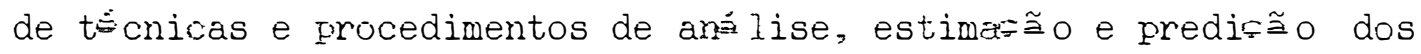
modelos de efeitos mistos, principalmente no que se relaciona com estruturas de dados desbalanceados.

SCHEFPé (1959) no seu livro clássico de Análise de Variância, busca esclarecer e unificar a teoria bá sica da Análise de Variância, desenvolvida até a referida data. Ali sầo estudados com mita clareza os modelos de efeitos aleatórios e mistos com estrutura desbalanceada, embora nada seja apresentado quanto aos preditores, fato justificável pois apenas na última década esse tema ten sido abordado com maior interesse.

HARVEY (1964) faz uso dos mínimos quadrados para desenvolver todo um procedimento aritmético que permite obter as estimativas de paràmetros, somas dos quadrados, esperancas dos quadrados nédios e estimativas de componentes de variância nos modelos mistos com mais de un fator de classificaç̃o. Ele só leva em consideracăo o modelo superparametrizado, e tanísen nada fala sobre os preditores. 
HARTLEY \& RAO (1967) a partir do modelo de efeitos mistos,

$$
Y=x a+u_{1} b_{1}+\cdots+U_{c} b_{c}+e
$$

e assumindo que $Y$ segue uma distribuicão normal multivariada com matriz de variâncias e covariâncias.

$$
\sigma^{2} H=\sigma^{2}\left[I_{n}+\gamma_{1} u_{1} u_{1}+\ldots+\gamma_{c} u_{c} u_{c}\right]
$$

onde $\gamma_{i}=\sigma_{i}^{2} / \sigma^{2} ;$ fizeram uso do mf́todo de estimaš̃o de máxima verossimilhanc a (ML) para estimar $\alpha, \sigma^{2}$ e $\gamma_{i}, i: 1, \ldots, c$, mas na estimacão dos $\gamma_{i}$ como não existe uma solucão implícita, desenvolveram um método numérico analítico para obter estas estimativas. Discutiram a eficiência e consistência assintótica dos estimadores e finalmente como trabalham sob normalidade, testaram hipóteses e construiram regiões de confianca. Este é um trabalho clássico na obtencão dessas estimativas.

HARTLEY (1967) partindo do modelo [2.11, desenvolve um procedimento geral para obter de modo direto os valores numéricos dos coeficientes associados aos quadrados médios esperados em alguns modelos mistos desbalanceados, assim como em algumas análises de variâncias, ou seja o método é restrito embora constitui uma ferramenta prática e simples, na obtencão desses coeficientes. A técnica por ele desenvolvida é aplicável ao caso dos modelos superparametrizados.

HARVEY (1970), apresenta procedimentos computacionais para estimar os componentes de variàncias e covariâncias, quando 
algum conjunto de efeitos aleatórios interage com um ou dois conjuntos de efeitos fixos e a estrutura dos dados é não balanceada. No seu trabalho faz uso dos mínimos quadrados no processo de estimacão, e também, só leva em consideracão modelos superparametrizados.

HARTLEY \& VAUGHN (1972), partindo do modelo [2.1], e sob normalidade com estrutura de covariâncias dada em [2.2], apresentam um desenvolvimento computacional para a solucão das equacões de máxima verossimilhama, baseado na solusão assintótica de c-equacões diferenciais. Esse método é conhecido como o de etapa ascendente. Mediante tal procedimento, é possível obter estimativas assintóticas de razões de variâncias as quais são de considerável importância nos estudos de herdabilidade genética.

HEMMERLE \& HARTLEY (1973), novamente a partir do modelo [2.1] e levando em consideracão [2.2], usam a transformacão W na obtencão dos estimadores de máxima verossimilhanca, tanto para os componentes fixos como para os componentes de variância. Essa tranformacão não só permite eliminar os cálculos explícitos e necessários na obtencão de $\mathrm{H}^{-1}$, como também desenvolver os cálculos de forma que não dependam do número de observacõos em qualquer das etapas. 0 método da transformacão W pode ser considerado como uma modificação do método de Newton-Raphson, no qual é imposta a restricão de que os componentes de variância sejam não negativos. Neste trabalho também apresentam a solucão dos componentes de variância usando o método de Newton-Raphson, assim como exemplos de comparacão entre os dois métodos.

SPEED \& HOCKING (1974), a partir do fato de que a 
determinacão dos coeficientes associados aos componentes de variância nos quadrados médios esperados é de grande interesse, desenvolveram um método numérico para a estimacão desses coeficientes e também dos componentes no caso de caselas com frequências desiguais. Um fato importante neste trabalho, foi que na solucão das somas de quadrados e suas esperancas, estabelecem uma analogia com o modelo de efeitos fixos, facilitando a obtencão dos resultados, além disso quando se faz uma reparametrização do modelo ou quando se usa o modelo de médias, o método por eles apresentado facilita muito a obtencão dos resultados.

CORBELL \& SEARLE (1976), novamente a partir do [2.1 1 e com a estrutura de variâncias-covariâncias [2.2], levando em consideração a normalidade, fizeram uma particão do modelo em duas partes, uma delas ficando livre dos efeitos fixos. A seguir ela é maximizada, obtendo-se assim o procedimento de estimacão conhecido como o de máxima verossimilhanc a restrita (REML). Esse método têm a vantagem, sobre o de máxima verossimilhansa, de que os estimadores REML, além de não serem invariantes apenas para os efeitos fixos no modelo, no caso balanceado, fornecem idênticos resultados aos obtidos pela Análise de Variância. Na solucão do sistema, desenvolveram um algoritmo adaptando a transformacão desenvolvida por HEMMERLE \& HARTLEY (1973), a qual reduzindo a dimensão dos cálculos e utilizando matrizes de dimensão igual à do espaco dos parâmetros, ao invés de matrizes de dimensão do espaco amostral. Apresentaram alguns exemplos onde são comparadas as estimativas obtidas através da ANOVA, ML e REML, tanto no caso balanceado como desbalanceado. Este trabalho também foi 
considerado um clássico na estimac̃ão dos componentes de variância. HENDERSON (1977), calcula as variâncias dos estimadores quadráticos não viesados dos componentes de variância mediante métodos de simulacão, examinando propriedades dos estimadores da variância e seus quocientes através da técnica de redũão dos mí nimos quadrados.

HARVEY (1979), apresentou o método do ajuste de constantes na análise de mínimos quadrados, aplicável nos seguintes casos:

a) Um critério de classificacão com e sem efeitos de covariá veis;

b) Dois critérios de classificacão com e sem interacão levando em consideração os efeitos de covariáveis;

c) Classificacão múltipla e cruzada e com efeitos de covariáveis.

O autor considerou os modelos superparametrizados e a estrutura de dados desbalanceados; apresentou várias alternativas na obtencão dos coeficientes associados às esperancas dos quadrados médios. Esse trabalho tem sido amplamente difundido entre pesquisadores do setor agropecuário.

GOODNIGHT \& SPEED (1980), calcularam as esperancas dos quadrados médios para a análise de variância associada com modelos não balanceados mistos, e calcularam os coeficientes desses quadrados médios esperados a partir de uma modificas̃a da técnica desenvolvida por SPEED \& HOCKING (1974). A modificação levou em conta a técnica da raiz quadrada ou de Cholesky na obtencão da matriz da forma quadrática associada à esperanca dos quadrados 
médios, no trabalho só consideram o caso de modelos superparametrizados.

HENDERSON JR. (1982), apresentou um resumo da estimacão e teste de hipóteses para a análise de covariância em modelos mistos, incluindo o caso de coeficientes aleatórios de regressão. Considerou também o estudo de covariàveis não homogêneas de regressão no modelo misto, no contexto da interpretação de futuras diferencas da predicão entre os ní veis de um fator determinado ou da interacão. Também examinou diferentes coeficientes aleatórios de regressão associados aos ní veis dos efeitos aleatórios.

O autor considerou que os coeficientes da regressão são estimáveis sempre que as colunas da matriz de delineamento das covariáveis, sejam linearmente independentes das colunas da matriz de delineamento para os ní veis dos fatores fixos e interacôes, a grande utilidade deste trabalho, consiste na análise feita sobre os modelos mistos com estruturas de covariáveis.

HENDERSON (1984 a,b), numa das obras clássicas de modelos lineares com efeitos aleatórios e mistos, mostrou uma clara tendência da sua utilizas̃ão na ciência animal, principalmente no melhoramento genético. Apresentou um conjunto de técnicas e métodos para a estimacão dos componentes de variância, e também do BLUE e do BLUP no caso do modelo superparametrizado com estruturas desbalanceadas e tamłém com caselas vazias. A clareza no desenvolvimento teórico, e as múltiplas aplicacôes apresentadas, constituiram grande contribuicão ao estudo desses modelos.

DODGE (1985), apresentou técnicas para a análise de 
informasões de dados obtidos de experimentos onde podem ocorrer caselas vazias. Argumentou que no caso de perda de parcela os delineamentos perdem sua simetria, e portanto, nem todas as funcöes paramétricas são estimáveis; levando a uma inferência inapropriada sob as hipóteses, e desse modo, a erros na conclusão dos resultados.

Para abordar o problema, o autor construiu a matriz de incidência $\mathrm{N}$ que fornece informaröes sob as funcöes estimáveis, introduz o algorí tmo conhecido como do R-processo, que permite determinar quais os valores esperados das caselas são estimáveis. O autor não considerou o caso dos modelos mistos e aleatórios, isto é, só considerou os modelos de efeitos fixos.

HOCKING (1985), trabalhou com os modelos de médias com estruturas desbalanceadas e ainda com caselas vazias. Embora dê maior ênfase aos modelos dos efeitos fixos, quando aborde os modelos de efeitos aleatórios e mistos, apresentou técnicas de obtencão dos componentes de variância usando estimativas de máxima verossimilhanca. Leva também a cabo testes de hipóteses usando a reparametrizacão encontrada em SPEED \& HOCKING (1974).

\section{GOLDSTEIN (1986) apresentou a técnica iterati}

va dos mínimos quadrados generalizados motivando o seu uso em muitas áreas. A grande vantagem do algorí tmo apresentado está na sua generalidade sobre outros métodos de estimacão como o de máxima verossimilhanca. No tocante à não exigência de uma distribuicão, sob normalidade os dois métodos fornecem resultados equivalentes na estimativa dos componentes de variància. O autor leva em consideracăo apenas 
modelos hierárquicos e além de estimar os componentes de variância mostrou que essas estimativas satisfizeram muitas propriedades da convergência. Discutiu também, a aplicacão do método dos mínimos quadrados iterativos restritos no caso de dados longitudinais a estimasão em modelos múltiplos multivariados, e caselas vazias, além de modelos com erros de medida nas variáveis independentes.

SEARLE (1987) trabalhou com modelos não balanceados e considerou que é importante estudar este tipo de estrutura de dados pelo seguinte:

a) a análise para o caso balanceado já tem sido amplamente estudada;

b) o estudo de dados desbalanceados vem sendo amplamente aprofundado, provavelmente pelo uso cada vez maior dos computadores;

c) a "assustadora" aritgética requerida na análise destes tipos de informasc̃es vem sendo facilitada pelo uso dos pacotes estatí sticos.

A maior parte da teoria apresentada levou em consideracão os modelos de médias e modelos de efeitos fixos, embora abordando modelos de efeitos mistos através de diferentes métodos de estimacão.

SEARLE (1988) fez uma apresentasão histórica dos modelos de efeitos aleatórios no período 1820-1947, além disso fez um desenvolvimento próprio dos métodos da máxima verossimilhansa e máxima verossimilhanca restrita na obtencão dos componentes de variância. O método esta baseado na obtencão dos estimadores de 
máxima verossimilhanca a partir de um subconjunto de dados, onde cada particão fez uso do método de ML, e depois simplesmente combinou o conjunto de estimadores.

Mas como nem sempre existem sub-conjuntos independentes, foram usados os pronédios na estimasão dos respectivos componentes respectivos.

\section{2. Resumo Histórico}

Segundo SCHEFṔ (1959) o nétodo de estimasão dos mínimos quadrados foi estudado de forma independente, e publicado em livros de problemás de Astronoñia por Legendre em 1806 e Gauss en 1809.

Conforme MENDEZ (1978) os modelos de efeitos aleatórios foram inicialmente estudados por Airy em 1861 que propôs um modelo linear com efeitos aleatórios muma aplicacão ná Astronomia. Chauvenet citado por MENDEZ, en 1863, faz uso dos resultados de Airy numa aplicacão à teoria de Bessel.

Segundo SEARLE (1988) os estudos mais recentes desses modelos encontram-se nos trabalhos de Fisher, que em 1918 publicou um artigo sobre genética quantitativa, onde utiliza:

i) os termos variânciá e análise dá variânciá;

ii) ná forma implícita o termo componentes de variância.

Em 1925 Fisher, citado por SEARLE, muito contribuiu à teoria dos componentes de variância quando apresentou a teoria da 
Análise da Variância (ANOVA), nesse estudo, prop̃̃e un método de estimasão dos componentes, igualando-se a soma dos quadrados da ANOVA ao seus quadrados médios esperados, obtendo assim um conjunto de equasões lineares que, solucionado, fornece estimativas dos componentes de variância.

Também nesta data, estendeu a Análise de Variância de um modelo de classificasão simples, desbalanceado, ao classificacão dupla (2 fatores) com interacão.

Continuando com SEAREE (1988), tem-se que Tippet em 1931 classifica e estende o nétodo de estimarão da ANOVA, agregando à tabela a coluna dos quadrados médios esperados, e diz que no caso de dados desbalanceados "não é possí vel obter a soma dos quadrados dos desvios dos grupos de médias com relacão a média geral, cada grupo vai ter diferentes ponderasões".

Nesse perí odo ainda não se concebia o estudo dos modelos mistos com dados desbalanceados como se vê em Tippet e também em Snedecor 1934, que diz, no caso de dados desbalanceados: "a relacão direta entre a análise de variância e a correlacão intraclasse desaparece se não houver igual frequência nas caselas".

Depois de 1947 o interesse pela estimacão dos componentes de variância foi estudado amplamente pelas múltiplas aplicacões com seres humanos, gado leiteiro, gado de corte, cereais, porcos e assim por diante. Em grande número de situasões a estrutura dos dados é não balanceada.

Um marco inicial significativo dos estudos de componentes de variâria no caso desbalanceado pode ser encontrado 
em HENDERSON (1953), que seguindo o método de igualar a soma dos quadrados com os seus valores esperados, iguala uma variedade de formas quadráticas com os seus valores esperados.

Apresenta três métodos dos quais o método III é o de maior interesse, pelo fato de que nos modelos mistos as estimativas dos componentes de variância não são afetados pelos efeitos fixos. Tem a desvantagem de envolver cálculos das inversas generalizadas que podem ser muito trabalhosos no caso de um grande número de fatores.

No perí odo 1967-1972 foram desenvolvidos três diferentes métodos considerados clássicos na estimarão dos componentes, os quais são:

a) de HARTLEY \& RAO (1967) que apresentaram o método da máxima verossimilhans a $(M L=M V)$;

b) de máxima verossimilhansa restrita (REML=MVR) apresentada inicialmente por ANDERSON \& BANCROFT (1952), extendida por PATTERSON \& THOMPSON (1971), e por CORBELL \& SEARLE (1976);

c) 0 método da mí nima norma quadrática MINQUE estudado simultaneamente por LA MOTTE (1973) e RAO (1971, 1972);

Com estes três métodos como base, muitos outros pesquisadores continuaram trabalhando nos estudos desses modelos, como pode ser observado na revisão de literatura. 


\section{RESULTADOS PRELIMINARES}

\subsection{Introdução}

Como este trabalho busca atingir basicanente os modelos lineares com efeitos aleatórios e mistos, e, considerando-se que os três aspectos básicos no estudo dos modelos mistos são:

i) estimacão dos efeitos fixos;

ii) estimacão dos efeitos aleatórios;

iii) obtencão da matriz inversa dos componentes de variância visando a obtencão da estimacão desses componentes.

Então nessa ordem se faz o desenvolvimento deste capí tulo.

\section{2. Modelo Matemático}

Seja o modelo Iinear

$$
\mathrm{N}_{1}=\mathrm{N}_{\mathrm{K}} \mathrm{B}_{1}+\mathrm{N}_{\mathrm{q}} \mathrm{Z}_{1}+\mathrm{N}_{1} \mathrm{e}_{1}
$$

y: vetor das observacổes com dimensões $\mathrm{Nx} 1$

$X$ : matriz conhecida associada aos efeitos fixos de dimensões $N \times K ; r[X] \leq \min \{N, K\}$

B: vetor de efeitos fixos desconhecidos de dimenseses $k \times 1$ 
u: vetor não observável de dimensões qx1

$$
q=\sum_{i=1}^{s} q_{i} ; u=\left[u_{1}, u_{2}, \ldots, u_{s}\right]
$$

$u_{i}:$ vetor aleatório de dimensão $q_{i} \times 1$

Z: é usualmente uma matriz de incidência associada aos efeitos aleatórios, com dimensões $\mathrm{Nxq} e$, em geral observá vel:

$$
Z=\left[z_{1}, z_{2}, \ldots, z_{s}\right]^{\prime}
$$

$Z_{i}:$ matriz observável de dimensões $\mathrm{Nxq}_{i}: i=1,2, \ldots$,

e: vetor não observável ou vetor de erros no modelo de dimensões $\mathrm{N} \times 1$

3.2.1. Valores esperados e matrizes de variâncias e covariâncias

As pressuposicões básicas sobre os valores esperados e variâncias e covariâncias adotadas neste estudo, são:

i) se $u_{0}=e$ então $u^{\prime}=\left[u_{0}^{\prime}, u_{1}^{\prime}, \ldots, u_{s}^{\prime}\right]$, e

$$
E\left(u_{i}\right)=0, \forall i=0,1, \ldots, s
$$

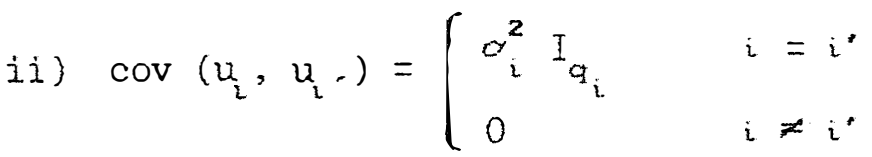

De [3.2] como $u_{0}=e$; então $\operatorname{Var}(e)=d_{e}^{2} I_{N}$ e também $\operatorname{cov}\left(u_{i}, e\right)=0 ; i=1, \ldots, s$.

iii) $\operatorname{Var}(y)=\operatorname{Var}(\mathrm{X} \beta+\mathrm{Zu}+\mathrm{e})=\mathrm{Z} \operatorname{Var}(\mathrm{u}) \mathrm{Z}^{\prime}+\operatorname{Var}(\mathrm{e})+\mathrm{Zcov}(\mathrm{u}, \mathrm{e})$ 
$=Z D^{\prime}+R=Z D Z^{\prime}+\sigma_{e}^{2} I_{N} ; R=\operatorname{Var}(e)+Z \operatorname{cov}(u, e)=\sigma_{e}^{2} I_{N}$ onde $D=\stackrel{s}{\stackrel{s}{\oplus}} \sigma_{i}^{2} I_{q_{i}} ; \quad$ soma direta de matrizes.

então

$$
\begin{aligned}
\operatorname{Var}(y) & =Z\left(_{i=1}^{s} \sigma_{i}^{2} I_{q_{i}}\right) Z^{\prime}+\sigma_{e}^{2} I_{N} \\
& =\sum_{i=0}^{s} Z_{i} D_{i} Z_{i}^{\prime}=\sum_{i=0}^{s} Z_{i} Z_{i}^{\prime} \sigma_{i}^{2} \\
& =Z D Z^{\prime}+R=V \\
\operatorname{cov}\left(y, u^{\prime}\right) & =E\left[(y-E(y))(u-E(u))^{\prime}\right] \\
& =\sum_{i=1}^{s} Z_{i}^{2} \sigma_{i}=Z D=C
\end{aligned}
$$

Neste caso $D_{i}=\sigma_{i}^{2} T_{q_{i}} \quad ; \quad i=0,1, \ldots, s$

então de forma conjunta é escrita:

$$
\operatorname{var}\left[\begin{array}{l}
\mathrm{y} \\
\mathrm{u} \\
\mathrm{e}
\end{array}\right]=\left[\begin{array}{lll}
\mathrm{V} & \mathrm{C} & \mathrm{R} \\
\mathrm{C}^{\prime} & \mathrm{D} & 0 \\
\mathrm{R} & \mathrm{O} & \mathrm{R}
\end{array}\right]
$$

\subsection{Estimacão dos Ef́eitos Fixos e Aleatórios}

Eelo fato de que o maior interesse no processo de estimacão do modelo [3.1], concentra-se nos melhores estimadores lineares não viesados (BLUES), os quais estão associados aos efeitos fixos do modelo, e os melhores preditores lineares não 
viesados (BLUPS), associados aos efeitos aleatórios, nesta seç̃̃o desenvolve-se a teoria pertinente, segundo as propostas deste estudo.

Seja o modelo [3.1] onde

$$
\begin{aligned}
& \text { e } n\left(0, \alpha^{2} R\right) \text {, ou em forma equivalente } \\
& E(e)=0, \operatorname{Var}(e)=R \alpha^{2}
\end{aligned}
$$

$R$ não singular, então existe uma matriz $A$ ortogonal tal que

$$
A A^{-}=R^{-1} \text {, }
$$

pré-multiplicando-se $[3.1]$ por $A^{\prime}$, tem-se:

$$
A^{\prime} y=A^{\prime} X / 3+A^{\prime} Z u+A^{\prime} e
$$

e então

$$
W=T \beta+F u+e^{*}
$$

onde

$$
W=A^{\prime} y ; T=A^{\prime} X ; F=A^{\prime} Z \text { desse modo, }
$$

$$
e^{*} n\left(0, \sigma^{2} I\right)
$$

Assim ao aplicar-se o método dos mínimos quadrados generalizados em [3.7], obtém-se

$$
Q=(W-T \beta-F u)^{\prime}(W-T \beta-F u)
$$

Igualando-se a zero as derivadas parciais

i) $\frac{\partial Q}{\partial \beta}=0$

ii) $\frac{\partial Q}{\partial u}=0$

Chega-se ao seguinte sistema

1) $X^{*} \cdot R^{-1} X \beta^{0}+X^{\prime} R^{-1} Z \hat{u}=X^{*} \cdot R^{-1} y$

[3.8]

2) $Z^{\prime} R^{-1} X \beta^{\circ}+\left(Z^{\prime} R^{-1} Z+D^{-1}\right) \hat{u}=Z^{\prime} R^{-1} y$ 
21.

donde, para o valor de û na equasão (2) de [3.8] tem-se:

$$
\hat{u}=\left(Z^{\prime} R^{-1} Z+D^{-1}\right)^{-}\left(Z^{\prime} R^{-1} y-Z^{\prime} R^{-1} X \beta^{\circ}\right)
$$

e ao substituir-se [3.9] na equačão (1) de [3.8], e usando-se em [3. 4] o fato de que segundo HENDERSON \& SEARLE (1981):

$$
(A+U \dot{U B V})^{-1}=A^{-1}-A^{-1} U\left(I+\dot{B V} A^{-1} U\right)^{-1} \dot{B V A} A^{-1}
$$

obtém-se:

$$
X^{\prime} V^{-1} X \beta^{\circ}=X^{\prime} V^{-1} Y
$$

donde

$$
\beta^{0}=\left(X^{\prime} V^{-1} X\right)^{-} X^{\prime} V^{-1} y
$$

É imediato verificar que [3.11] satisfaz

a) $E\left(\beta^{\circ}\right)=E\left[\left(X^{\prime} V^{-1} X\right)^{-} X^{*} V^{-1} y\right]=\beta$

b) $\operatorname{BLUE}\left(X \beta^{\circ}\right)=X\left(X^{\prime} V^{-1} X\right)^{-} X^{\prime} V^{-1} y$

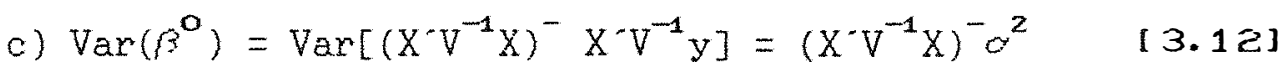
onde $\left(\mathrm{X}^{\top} \mathrm{V}^{-1} \mathrm{X}\right)^{-}$é uma inversa generalizada de $\left(\mathrm{X}^{\top} \mathrm{V}^{-1} \mathrm{X}\right)$

d) Quando a matriz associada aos efeitos fixos é de posto coluna completa, então pode-se obter uma matriz B da forma

$$
B=\left(X^{\prime} V^{-1} X\right)^{-1} X^{\prime} X
$$

com a qual [3.11] é equivalente à solucão dos mínimos quadrados ordinários (M.Q.O.).

Prova: Se B é não singular então

$$
V X=X B
$$

i) pela não singularidade de $B$, vai existir $B^{-1}$, de tal maneira que pré-multiplicando por $\mathrm{V}^{-1}$ e pós-multiplicando por $\mathrm{B}^{-1} e$ 
transpondo, [3.13] passa a ser dado por

$$
X^{\prime} V^{-1}=\left(B^{\prime}\right)^{-1} X^{\prime}
$$

que pós-multiplicando por $\mathrm{X}$, e invertendo tem-se

$$
\left(X^{\prime} V^{-1} X\right)^{-1}=\left(X^{*} X\right)^{-1} B^{\prime}
$$

mas

$$
B^{\prime}=X^{\prime} V X^{\prime-1} \Rightarrow\left(X^{\prime} V^{-1} X\right)^{-1}=\left(X^{\prime} X\right)^{-1}\left[X^{\prime} V X^{\prime-1}\right]
$$

e após multiplicar por $X^{\prime} V^{-1}$ então

$$
\begin{aligned}
& \left(X^{\prime} V^{-1} X\right)^{-1} X^{\prime} V^{-1}=\left(X^{\prime} X\right)^{-1} X^{\prime} V X^{\prime}{ }^{-1} X^{\prime} V^{-1} \\
\Rightarrow \quad & \left(X^{\prime} V^{-1} X\right)^{-1} X^{\prime} V^{-1}=\left(X^{\prime} X\right)^{-1} X^{\prime} \\
\Leftrightarrow \quad & \left(X^{\prime} V^{-1} X\right)^{-1} X^{\prime} V^{-1} Y=\left(X^{\prime} X\right)^{-1} X^{\prime} y \\
\Leftrightarrow \quad & \beta_{M Q G}^{\circ}=\beta_{M Q O}^{\circ}
\end{aligned}
$$

Para ver a equivalência, se [3.16] é satisfeita, então em [3.15] ao pré-multiplicar por $X^{\prime} X$ e pós-multiplicar por $V$ obtém-se que $\mathrm{VX}=\mathrm{XB}$, com o qual fica demonstrado o teorema.

\section{Observacöes:}

a) Se em [3.4] $Z_{o} \neq I_{N} \Leftrightarrow R \neq \sigma^{2} I_{N}$, então $V$ é singular, assim de [3.1] tem-se que $X \beta^{\circ}=X\left(X^{\prime} V^{-} X\right)^{-} X^{\prime} V^{-} y, j a ́$ não vai satisfazer a propriedade de ser um estimador não viesado.

b) Se unN(O, D) e, enN(O, $\left.\sigma_{e}^{2} I_{N}\right)$ então $X / 3^{\circ}$ obtido por mí nimos quadrados generalizados é igual à solucão de máxima verossimilhanca - Ver HARTLEY \& RAO (1967) e a seção [4.e] deste trabalho. 


$$
\begin{aligned}
\hat{u} & =\left(Z^{\prime} R^{-1} Z+D^{-1}\right)^{-}\left(Z^{\prime} R^{-1} y-Z^{\prime} R^{-1} X\left(X^{\prime} V^{-1} X\right)^{-} X^{\prime} V^{-1} y\right] \\
& =\left(Z^{\prime} R^{-1} Z+D^{-1}\right)^{-} Z^{\prime} R^{-1}\left(y-X \beta^{0}\right)
\end{aligned}
$$

Em HENDERSON (1984, a) prova o resultado:

$$
\text { se } K=Z D \text { então } K^{*} V^{-1}=D^{\prime} Z^{\prime} V^{-1}
$$

ao substituir-se $\mathrm{V}^{-1}$ como em [3.10] e depois de somando-se e subtraindo-se a quantidade $D^{-1}\left(Z^{\prime} R^{-1} Z+D^{-1}\right) Z^{\prime} R^{-1}$ tem-se então que $K^{\prime} V^{-1}=D^{\prime} Z^{\prime} V^{-1}=\left(Z^{\prime} R^{-1} Z+D^{-1}\right)^{-} Z^{\prime} R^{-1}$.

Sendo assim, chega-se a

$$
\hat{u}=K^{\prime} V^{-1}\left(y-X \beta^{\circ}\right)
$$

que é equivalente a [3.17]

Pode-se observar que

$$
\left[\begin{array}{l}
y \\
u
\end{array}\right] \cap\left[\left(\begin{array}{l}
x \beta \\
0
\end{array}\right] ;\left(\begin{array}{ll}
v & C \\
C^{\prime} & D
\end{array}\right]\right]
$$

então [3.18] é tido como o melhor preditor para u. Desta, vê-se claramente que quando se leva em consideracão [ 3.19 então

$$
\hat{u}=E(u \mid y)
$$

observações: Do resultado obtido em [3.18] tem-se que:

i) $E(\hat{u})=0$

ii) $V(\hat{u})=D Z^{\prime} V^{-1} Z D-D Z^{\prime} V^{-1} X\left(X^{\prime} V^{-1} X\right)^{-} X^{\prime} V^{-1} Z D=$

$$
=K^{\prime} V^{-1}\left[I-\operatorname{Var}\left(X \beta^{0}\right) V^{-1}\right] K
$$

iii) Pelo fato de que $x / 3^{\circ}$ é invariante $\forall / 3^{\circ}$, a solucão do sistemá [3.8], então û têm solusãa única. 


\section{4. Caracterização dos Preditores Lineares}

Seja una funcão linear das observasões $L^{\prime} y$, conhecida como o preditor e que pode ser expressa como uma funsão linear de $N^{\prime} B+M^{\prime} u$ conhecido como o preditante, ou seja

$$
L^{\prime} y=N^{\prime} B+M^{\prime} u_{*}
$$

busca-se obter una estinativa do preditor, tal que seja mí nimo o erro de predicão, então procura-se ninimizar a variânia do erro de predicão.

Pelo fato de que

$$
E\left[L^{\prime} y\right]=L^{\prime} X \beta=N^{\prime} \beta
$$

onde $\mathrm{N}^{\prime}=L^{\prime} \mathrm{X}$ é estinável, então conforme IEMA (1987) minimiza-se a variancia do erro de predicão a qual é dada por

$\operatorname{Var}\left[N^{\prime} \beta+M^{\prime} u-L^{\prime} y\right]=\operatorname{Var}\left(N^{\prime} \beta+M^{\prime} u-L^{\prime} y\right]$

$$
=M^{\prime} D M+L^{\prime} V L-M^{\prime} D Z L-L^{\prime} Z D M
$$

sujeita a condisão $\quad N^{\prime}=L^{\prime} X$

Levando-se em consideração [ 3.22], obtém-se a função lagrangeana:

$$
Q=M^{\prime} D M+L^{\prime} V L-M^{*} D Z L-L^{\prime} Z D M+\left(L^{\prime} X-N^{\prime}\right) \lambda
$$

diferenoiando-a em relacão aos parânetros desconhecidos $(L, \lambda)$, chega-se ao sistema

$$
\left[\begin{array}{ll}
\mathrm{V} & \mathrm{X} \\
\mathrm{X}^{\prime} & \theta
\end{array}\right]\left[\begin{array}{l}
\mathrm{L} \\
\theta
\end{array}\right]=\left[\begin{array}{c}
\mathrm{ZDM} \\
\mathrm{N}
\end{array}\right]
$$


onde

$$
\theta=\frac{1}{2} \lambda \text { e } \quad \mathrm{V}=\mathrm{ZDZ}+\mathrm{R}
$$

Nota-se que

$$
\begin{aligned}
& \mathrm{VL}+\mathrm{X} \Theta=\mathrm{ZDM} \\
& \Leftrightarrow \quad\left(Z D Z^{\prime}+R\right) L+X \ominus-Z D M=0 \\
& \Leftrightarrow \quad Z D\left(Z^{\prime} L-M\right)+R L+X \Theta=0 \quad[3.25] \\
& \text { ao fazer } D\left(Z^{\prime} L-M\right)=A \Rightarrow M=Z^{\prime} L-D^{-1} A
\end{aligned}
$$

Ao substituir-se [3.25] e [3.26] em [3.24] tem-se o seguinte sistema

$$
\left[\begin{array}{ccc}
R & Z & X \\
Z^{\prime} & -D^{-1} & 0 \\
X^{\prime} & 0 & 0
\end{array}\right]\left[\begin{array}{l}
L \\
A \\
\theta
\end{array}\right]=\left[\begin{array}{l}
0 \\
M \\
N
\end{array}\right]
$$

Da primeira equação do sistema [ 3.27] tem-se

$$
L=-R^{-1}[Z A+X \ominus]=-R^{-1}[Z ; X] \quad\left[\begin{array}{l}
A \\
\theta
\end{array}\right]
$$

que ao substituir-se nas equacôes [2] e [ 3] do mesmo sistema [ 3.28], obtendo-se

$$
-\left[\begin{array}{ll}
Z^{\prime} R^{-1} Z+D^{-1} & Z^{\prime} R^{-1} X \\
X^{\prime} R^{-1} Z & X^{\prime} R^{-1} X
\end{array}\right] \cdot\left[\begin{array}{l}
A \\
\theta
\end{array}\right]=\left[\begin{array}{l}
M \\
N
\end{array}\right]
$$

Tem-se $-S\left[\begin{array}{l}A \\ \theta\end{array}\right]=\left[\begin{array}{l}M \\ N\end{array}\right]$; donde $\left[\begin{array}{l}A \\ \theta\end{array}\right]=S^{-}\left[\begin{array}{c}-M \\ -N\end{array}\right]$

De [3.30] e [3.28] obtém-se que

$$
L=-R^{-1}[Z, X] S^{-}\left[\begin{array}{l}
-M \\
-N
\end{array}\right]
$$




$$
L^{\prime}=\left[M^{\prime}, N^{*}\right] S^{-}\left[\begin{array}{l}
Z^{-} \\
X^{\prime}
\end{array}\right] R^{-\frac{1}{2}}
$$

e claramente

$$
L^{\prime} y=\left[M^{\prime}, N^{\prime}\right]\left[\begin{array}{ll}
Z^{\prime} R^{-1} Z+D^{-1} & Z^{\prime} R^{-1} X \\
X^{\prime} R^{-1} Z & X^{\prime} R^{-1} X
\end{array}\right]^{-1}\left[\begin{array}{l}
Z^{\prime} R^{-1} y \\
X^{\prime} R^{-1} y
\end{array}\right]
$$

e da solucão do sistema [3.8] tem-se que

$$
\hat{L^{\prime} y}=\left(M^{\prime}, N^{\prime}\right)\left[\begin{array}{l}
\hat{u} \\
\beta^{0}
\end{array}\right]=M^{*} \hat{u}+N^{\prime} \hat{\beta}^{\circ}
$$

Nota-se então que o preditor linear das observarões depende linearmente das estimativas de $\hat{\beta}^{\bullet}$ e de $\hat{u}$.

Supondo agora a relaç̃o linear

$$
\hat{H}=L^{\prime} y+b
$$

onde b é uma constante, combinasão linear dos elementos de y, com $\hat{H}=\hat{L^{\prime} y}+\hat{b}$, onde $\hat{u}$ e $\hat{b}$ são obtidos ao minimizar-se o erro

$$
\begin{aligned}
E[\hat{H}-H]^{2} & =E\left[L^{\prime} y+b-H\right]^{2} \\
& =E\left[\left(L^{\prime} y+b-H\right)^{\prime}\left(L^{\prime} y+b-H\right)\right] \\
& =E\left[\left(L^{\prime} y\right)^{2}+b^{2}+H^{2}+2 E\left(L^{\prime} y b\right)+2 L^{\prime} y H-2 b H\right] \\
& =L^{\prime} E\left(y^{2}\right) L+E\left(b^{2}\right)+E\left(H^{2}\right)+2 L^{\prime} E(y) b- \\
& -2 L^{\prime} E(y H)-2 b E(H)
\end{aligned}
$$

Por outro lado já é conhecido que:

i) $\operatorname{Var}(y)=V \Rightarrow E\left(y^{2}\right)=V+(X \beta)(X /)^{\prime}=V+\alpha \alpha^{\prime}$

ii) Se assumir $E(H)=\theta \Rightarrow E(H)^{2}=\operatorname{var}(H)+\theta^{2}$

iii) $\operatorname{cov}(y, H)=C \Rightarrow E(y H)=C+X \beta \theta=C+\alpha \Theta$ 
Ao substituir i, ii, iii em [3.34] tem-se a funcão a minimizar:

$$
\begin{aligned}
& Q=L^{\prime} V L+L^{\prime} a a^{\prime} L+b^{2}+\theta^{2}+ \\
& +\operatorname{var}(H)+2 L^{\prime} a b-2 L^{\prime} c-2 L^{\prime} a \theta-2 b \theta
\end{aligned}
$$

Ao obter as derivadas:

i) $\frac{\partial Q}{\partial L}=0$

ii) $\frac{\partial Q}{\partial b}=0$

gera-se o sistema de equasóes:

1) $\mathrm{LV}+\mathrm{La \alpha ^{ \prime }}+\mathrm{ab}=\mathrm{C}+\mathrm{a} \theta$

2) $b+L^{\prime} a=\theta$

com as soluções

$$
L=V^{-1} C \quad \text { e } \quad b=\theta-a^{\prime} V^{-1} C
$$

portanto

$$
\begin{aligned}
\hat{H} & =L^{\prime} y+b=\theta+C^{\prime} V^{-1}(y-\alpha) \\
& =E(H)+C^{\prime} V^{-1}(y-X \beta)
\end{aligned}
$$

Este resultado é conhecido como o melhor preditor linear em termos dos elementos de y (BLUP). Nota-se que é melhor preditor no sentido de que tem mí nima variância.

observacão: Se conjuntamente $y$ e $H$ se têm distribuicão multinormal, ou seja

$$
\left[\begin{array}{l}
y \\
H
\end{array}\right] \cap N\left[\begin{array}{l}
X \beta \\
\theta
\end{array}\right] ;\left(\begin{array}{cc}
V & C \\
C^{\prime} & \operatorname{var}(H)
\end{array}\right)
$$


então a distribuirão condicional de $\mathrm{H}$ dado y é tal que

$$
H \mid y \cap N\left[\theta+C^{\prime} V^{-1}(y-X \beta) ; \operatorname{var}(H)\right]
$$

Desse modo

$$
E(H \mid y)=\theta+C^{\prime} V^{-1}(y-X \beta)=\hat{H}
$$

da equação [3.36]

Fato: Sob normalidade [3.20] e [3.36] coincidem.

observąão: Qualquer que seja a distribuicão em [3.20] e [3.36] sempre é possível encontrar os momentos finitos de primeira é segunda ordem.

Pode-se observar que tanto o BLUE de

$$
X / 3^{\circ}=X\left(X^{\prime} V^{-1} X\right)^{-} X^{\prime} V^{-1} y,
$$

como o

$$
\operatorname{BLUP}(\hat{u})=C^{\prime} V^{-1}\left(y-X_{i} \beta^{\circ}\right)
$$

ou mesmo

$$
\operatorname{BLUP}(\hat{H})=\theta+C^{\prime} V^{-1}\left(y-X \beta^{\bullet}\right)
$$

dependem da matriz de variâncias é covariâncias $\mathrm{V}$, através de $\mathrm{V}^{-1}$, sendo assim considera-se importante apresentar neste trabalho algumas formas explícitas para sua obtencão. A forma apresentada permite estruturar em forma imediata a matriz $\mathrm{V}$ e portanto também $\mathrm{V}^{-1}$. 


\subsection{Sobre a obtenção de $\mathrm{v}^{-1}$}

O cálculo da matriz $\mathrm{V}^{-1}$ pode complicar-se, se na notacão de [3.4], a estrutura de $R$ for de natureza complexa, mas neste trabalho isto não acontece, pelo fato de que $R=c^{2} I_{N}$.

Inicialmente apresenta-se uma adaptasão do trabalho de SEARLE \& HENDERSON (1979) na obtencão de $\mathrm{V}^{-1}$ para o caso dos modelos superparametrizados e estrutura de dados balanceados quando $V=\Sigma \sigma_{i}^{2} Z_{i} Z_{i}^{\prime}$, então cada matriz $Z_{i} Z_{i}^{\prime}$, pode ser escrita como o produto Kronecker de matrizes $\mathrm{I}_{\mathrm{s}}$ e $\mathrm{J}_{\mathrm{s}}$ onde:

i) $I_{s}$ : matriz identidade de ordem $s$.

ii) $J_{s}$ : matriz quadrada de ordem $s$, com elementos todos iguais a uns.

iii) $I_{s 1} \otimes I_{s 2} \otimes I_{s 3}=I_{s 1 s 2 s 3}$

iv) $J_{s 1} \otimes J_{s 2}=J_{s 1 s 2}$

v) $\quad\left(J_{s}\right)^{0}=I_{s}$

Ao obter-se o modelo com a variável respesta,

$y_{i j k l . .1} ; i=1, \ldots, I ; j=1, \ldots J, \ldots, t=1, \ldots, T$

e se $\mathbf{P}$ representa o número total de sub-índices associados a variável resposta, então pode-se obter $2^{P}$ possí veis particóes de produtos de Kronecker com matrizes $\mathrm{J}_{s}$. $\mathrm{I}_{\mathrm{s}}$, como em IEMM(1985):

$$
\begin{aligned}
\Delta= & {\left[I_{I} \otimes I_{J} \otimes \ldots \otimes I_{T} ; I_{I} \otimes I_{J} \otimes \ldots \otimes J_{T} ; \ldots ;\right.} \\
& \left.J_{I} \otimes J_{J} \otimes \ldots \otimes J_{T}\right]
\end{aligned}
$$

onde cada elemento do vetor, pole ser expresso nos termos das 
variáveis binárias:

$$
\delta=\left(\begin{array}{l}
1 \text { se ocorrer } J_{s} \\
0 \text { se ocorrer } I_{s}
\end{array}\right.
$$

então [3. 39] é equivalente a

$$
\Delta_{1}=(00 \ldots 0 ; 00 \ldots 1 ; \ldots ; 11 \ldots 1)
$$

e com a notacão apresentada en [3.40], pode-se construir o vetor de componentes de variância estabelecendo-se una relacão un a um entre os sub-índices do componente aleatório do modelo e o zero (0) na igualdade $[3.40]$, e considerando zero como caso contrário. Obtem-se assim o vetor $\Theta_{\mathbf{1 \times 2}} \mathbf{P}$. Então

$$
V=\Delta \theta^{\prime}
$$

A seguir considera-se o seguinte produto de Kronecker

$$
T_{P}=\left[\begin{array}{ll}
1 & 0 \\
1 & I
\end{array}\right] \otimes\left[\begin{array}{ll}
1 & 0 \\
1 & J
\end{array}\right] \otimes\left[\begin{array}{ll}
1 & 0 \\
1 & K
\end{array}\right] \otimes \ldots \otimes\left[\begin{array}{ll}
1 & 0 \\
1 & T
\end{array}\right]
$$

Pelo fato de que

$$
\mathrm{T}_{\mathrm{P}}^{-1}=\left[\begin{array}{ll}
1 & 0 \\
1 & \mathrm{I}
\end{array}\right]^{-1} \otimes\left[\begin{array}{ll}
1 & 0 \\
1 & J
\end{array}\right]^{-1} \otimes \ldots \otimes\left[\begin{array}{ll}
1 & 0 \\
1 & T
\end{array}\right]^{-1}
$$

e se $\lambda_{\mathbf{P}}=\mathrm{T}_{\mathbf{P}} \theta_{\mathbf{P}}$ então

$$
V^{-1}=T_{P}^{-1} \lambda_{P}^{-1} \Delta^{\prime}
$$

observação: em muitos casos, é necessário que se faca permutacões no $\Delta$ e no $\theta$ para se obter $V$

Como uma aplicação:

Seja o modelo 


$$
\begin{gathered}
y_{i, j}=\mu+\alpha_{i}+\beta_{j}+\gamma_{i j}+\varepsilon_{i, j k} \\
i=1, \ldots, I ; j=1, \ldots, J ; k=1, \ldots, k
\end{gathered}
$$

Note que se, por exemplo $\mathrm{p}=3$ então $2^{3}=8$ particóes

$$
\Delta=\left[J_{I} \otimes J_{J} \otimes J_{K} ; J_{I} \otimes J_{J} \otimes I_{K} ; \ldots ; I_{I} \otimes I_{J} \otimes I_{K}\right]
$$

se em [3.43] apenas $\mu$ for fixo, então a relacão entre os sub-índices das conponentes aleatórias do modelo e as variáveis binárias ficám

$$
\begin{gathered}
\Delta_{1}=(111,110,101,011,100,010,001,000) \\
\Rightarrow \quad \theta=\left(0,0, \sigma_{\beta}^{2}, \sigma_{\alpha}^{2}, 0,0, \sigma_{\gamma}^{2}, \sigma_{e}^{2}\right) \\
T_{3}=\left[\begin{array}{ll}
1 & 0 \\
1 & I
\end{array}\right] \otimes\left[\begin{array}{ll}
1 & 0 \\
1 & J
\end{array}\right] \otimes\left[\begin{array}{ll}
1 & 0 \\
1 & K
\end{array}\right]
\end{gathered}
$$

e

Ao permutar $\Delta$, na forma

$$
\Delta=\left[I_{I} \otimes I_{J} \otimes I_{K} ; \ldots ; J_{I} \otimes J_{J} \otimes J_{K}\right]
$$

e $\theta=\left(\sigma^{2} e, \sigma_{\gamma}^{2} ; 0,0 ; \alpha_{\alpha}^{2} ; \sigma_{\beta}^{2}, 0,0\right)$, então 
32.

$$
\lambda_{3}=T_{3} \theta^{\prime}=\left[\begin{array}{l}
\sigma_{e}^{2}=\lambda_{0} \\
\sigma_{e}^{2}+K \sigma_{\gamma}^{2}=\lambda_{\alpha \beta} \\
\sigma_{\theta}^{2} \\
\sigma_{\theta}^{2}+K \sigma_{\gamma}^{2}+J K \sigma_{\alpha}^{2}=\lambda_{\alpha} \\
\sigma_{\theta}^{2} \\
\sigma_{e}^{2}+K \sigma_{\gamma}^{2}+I K \sigma_{\beta}^{2}=\lambda_{\beta} \\
\sigma_{\theta}^{2} \\
\sigma_{\theta}^{2}+K \sigma_{\gamma}^{2}+J K \sigma_{\alpha}^{2}+I K \sigma_{\beta}^{2}=\lambda_{\mu}
\end{array}\right]
$$

e então de [3.41], chega-se

$V_{3}=\Delta \theta^{\prime}=I_{X J K} \sigma_{e}^{2}+I_{I J} \otimes J_{K} \sigma_{\gamma}^{2}+I_{I} \otimes J_{J K} \sigma_{\alpha}^{2}+J_{I} \otimes I_{J} \otimes J_{K} \sigma_{\beta}^{2}$

e ao aplicar [3.42] tem-se então que

$V_{3}^{-1}=\frac{1}{\sigma_{e}^{2}} I_{I J K}-\frac{\sigma_{\gamma}^{2}}{\sigma_{e}^{2}\left(\sigma_{e}^{2}+K \sigma_{\gamma}^{2}\right)} \cdot\left(I_{I J} \otimes J_{K}\right)-$

$-\frac{\sigma_{\alpha}^{2}}{\left[\left(\sigma_{e}^{2}+K \sigma_{\gamma}^{2}\right)\left(\sigma_{e}^{2}+K \sigma_{\gamma}^{2}+J K \sigma_{\alpha}^{2}\right)\right]} \cdot\left(I_{I} \cdot J_{J K}\right)-$

$[3.45]$

$-\frac{\sigma_{\beta}^{2}}{\left[\left(\sigma_{e}^{2}+K \sigma_{\gamma}^{2}\right)\left(\sigma_{e}^{2}+K \sigma_{\gamma}^{2}+I K \sigma_{\beta}^{2}\right)\right]} \cdot\left(J_{I} \otimes I_{J} \otimes J_{K}\right)+$

$$
+\frac{K \sigma_{\alpha}^{2} \sigma_{\beta}^{2}\left\{\left(\sigma_{e}^{2}+K \sigma_{\gamma}^{2}\right)+\left(\sigma_{e}^{2}+K \sigma_{\gamma}^{2}+J K \sigma_{\alpha}^{2}+I K \sigma_{\beta}^{2}\right)\right\}}{\left(\sigma_{e}^{2}+K \sigma_{\gamma}^{2}+I K \sigma_{\beta}^{2}\right)\left(\sigma_{e}^{2}+K \sigma_{\gamma}^{2}\right)\left(\sigma_{e}^{2}+K \sigma_{\gamma}^{2}+J K \sigma_{\gamma}^{2}\right)\left(\sigma_{e}^{2}+K \sigma_{\gamma}^{2}+J K \sigma_{\alpha}^{2}+I K \sigma_{\beta}^{2}\right)} \cdot J_{I J K}
$$

observação: se $k=1$ e $\gamma=0$, então, tanto em [3.44] como em [3.45] é fácil identificar o modelo com dois fatores sem interacão 


$$
y_{i j}=\mu+\alpha_{i}+\beta_{j}
$$

quando apenas $\mu$ é fixo.

No caso em que a estrutura los dados for não balanceada, - desenvolvimento formal na inversão de $V$ depende do fato de que as componentes do modelo sejam cruzadas ou hierárquicas.

Assim por exemplo se no modelo

$$
\begin{gathered}
y_{i j}=\mu+a_{i}+\varepsilon_{i j}, \\
i=1, \ldots, a ; \quad j=1, \ldots, n_{i j} ; \quad N=\sum_{i=1}^{c} n_{i} .
\end{gathered}
$$

então

$$
\left.V=\sigma_{\theta}^{2} I_{N}+\sigma_{\alpha}^{2} I_{\alpha} \otimes J_{n_{i}}=\mathbf{H}_{e}^{2} I_{n_{i}}+\sigma_{\alpha}^{2} J_{n_{i}}\right\}
$$

nota-se que esta matriz pode ser escrita:

$$
\begin{gathered}
V=\stackrel{\leftrightarrow}{=1}_{e}^{a}\left(\omega_{n_{i}}^{2}+\sigma_{\alpha}^{2} I_{n_{i}} I_{(1)} I_{n_{i}}\right) \\
={ }_{i=1}^{\infty} V_{i} \text {, onde } 1_{r_{i}} \text { é o vetor unitário }
\end{gathered}
$$

Ao aplicar-se [3.10] no processo da inversão en

$$
\text { [3.46], } A=\sigma_{\theta}^{2} I_{n_{i}} ; \dot{V B V}=1_{n_{i}} I_{(1)} I_{n_{i}}^{\prime} \text { ten-se }
$$

que

$$
V^{-1}=\underbrace{a}_{i=1} \frac{1}{\sigma_{e}^{2}\left(\sigma_{e}^{2}+n_{i} \sigma_{\alpha}^{2}\right)}\left[\sigma^{2} I_{n_{i}}+D\left(n_{i} \sigma_{\alpha}^{2}\right)-\sigma_{\alpha}^{2} J_{i}\right]
$$

onde $D(a)$ : matriz diagonal de elementos iguais a a.

Agora para o nodelo hierárquico

$$
y_{i j k}=\mu+\alpha_{i}+\beta_{j(i)}+e_{i j k}
$$




$$
i=1, \ldots, a ; j=1, \ldots, b ; k=1, \ldots, n_{2 j}
$$

tem-se que para o i-ésimo nível do fator $\alpha_{i}$, a matriz de variâncias e covariâncias é dada por

$$
V_{i}^{*}=\sigma_{\theta}^{2} I_{r_{i}}+\sigma_{\alpha}^{2} J_{n_{i}}+\sigma_{\beta}^{2} D\left(J_{n_{i j}}\right)
$$

mas

$$
J_{n_{i, j}}=\left(1_{n_{i, j}} I_{(1)} 1_{n_{i, j}}^{\prime}\right)
$$

e

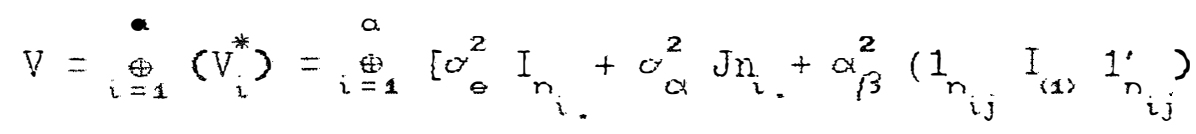

e por [3.47] tem-se que

$$
V=\underset{i=1}{\stackrel{\oplus}{\oplus}}\left[V_{i}+\sigma_{\beta}^{2}\left(1_{n_{i j}} I_{(1)} 1_{n_{i, j}}^{\prime}\right]\right]
$$

onde $V_{i}=\sigma_{\theta}^{2} I_{n_{i}}+\sigma_{a}^{2} J n_{i}$

e ao aplicar-se novamente [3.10] obtém-se a inversa da matriz V. De forma semelhante pode-se estender para o caso de modelos multihierárquicos.

Observação: No caso de modelos com fatores cruzados este procedimento não é válido. Deve-se então considerar casos especí ficos de inversão levando-se em consideração o número de componentes do modelo. 
3.6. Formas Explicitas do BLUE do BLUP nos Modelos com Um ou Dois Critérios de Classificação

Nesta sec̃̃o, apresentam-se algumas solusões particulares dos estimadores e preditores lineares nos modelos com un e dois critérios de classificasão, nos casos de estruturas de dados balanceados e desbalanceados. O objetivo é facilitar o uso destes resultados através do conhecimento de sua forma explícita.

Seja o modelo

$$
\begin{gathered}
y_{i j}=\mu+a_{i}+e_{i j} \\
\dot{i}=1, \ldots, a ; \quad j=1, \ldots, b ;
\end{gathered}
$$

onde apenás $u$ é fixo, por [3.41] tem-se que

$$
V=\sigma_{\theta}^{2} I_{n}+\sigma_{\alpha}^{2} I_{a} \otimes J_{b}=\sigma_{e}^{2} I_{n}+\sigma_{\alpha}^{2} D_{a}\left(J_{b}\right)
$$

onde

$$
I_{n}=I_{a} \cdot I_{b} ; n=a b
$$

e por [3.42], então

$$
V^{-1}=\frac{1}{\sigma_{e}^{2}}\left[I_{n}-\frac{\sigma_{a}^{2}}{\sigma_{\theta}^{2}+b \sigma_{a}^{2}} \stackrel{\oplus}{=}_{1} J_{b}\right]
$$

e assim por $[3.11]$ e por $[3.16]$ com $X=1_{r}^{\prime}=1^{\prime}$

$$
\hat{\mu}=\left(1^{\prime} V^{-1} 1\right)^{-1} 1^{*} V^{-1} y=(1 \cdot 1)^{-1} 1^{\prime} y=\bar{y} .
$$

Agora para obter a forma expli cita do BLUP, neste

caso para $\alpha$, então por [3.18] quando $V(\alpha)=\sigma_{\alpha}^{2} I_{\alpha}$ ten-se que $\operatorname{BLUP}(\hat{\alpha})=D\left[\frac{\alpha_{\alpha}^{2}}{\alpha_{e}^{2}+b \sigma_{\alpha}^{2}}\left(y_{i}-b_{i} \dot{\alpha}\right)\right]$ 


$$
=\frac{\sigma_{\alpha}^{2}}{\sigma_{e}^{2}+k \alpha_{\alpha}^{2}}\left[\begin{array}{ccc}
\left(y_{1}-\hat{b j \mu}\right) & 0 & 0 \\
0 & \left(y_{2}-\hat{b \mu}\right) & 0 \\
\vdots & \vdots & \vdots \\
0 & & \left(y_{a}-\hat{b \mu}\right)
\end{array}\right]
$$

Para um valor particular tem-se

$$
\operatorname{BLUP}\left(\alpha_{i}\right)=\frac{\alpha_{\alpha}^{2}}{\sigma_{e}^{2}+b \sigma_{\alpha}^{2}}\left(y_{i}-\hat{b u}\right)
$$

Agora se em [3.50] tem-se uma estrutura de dados näo balanceados, ou seja quando $J=1,2, \ldots, n_{\imath}$ então de $[3.11]$ e [3. 48 ] obtém-se

$$
\hat{\omega}=\operatorname{BLUE}(\mu)=\sum_{i}^{a} \frac{n_{i} \bar{y}_{i}}{\sigma_{e}^{2}+n_{i} \sigma_{\alpha}^{2}} / \sum_{i=1}^{a} \frac{n_{i}}{\sigma_{e}^{2}+n_{i} \sigma^{2}}
$$

Assim, de $[3.18]$ e [3.48] tem-se que

$$
\operatorname{BLUP}\left(\alpha_{i}\right)=\frac{\sigma^{2}}{\sigma_{\theta}^{2}+n_{i} \sigma_{a}^{2}}\left(y_{i}-n_{i} \hat{u}\right)
$$

Supondo agora que tem-se o modelo caracterizado por

$$
\begin{aligned}
& y_{i j k}=\mu+\alpha_{i}+\beta_{j}+e_{i j k} \\
& i=1, \ldots, a ; \quad j=1, \ldots, b ; \quad k=1
\end{aligned}
$$

Este modelo caracteriza os blocos completos ao acaso, onde o efeito dos blocos é aleatório, e além disso se na matriz associada aos efeitos considerar-se uma reparametrizacão, de tal forma que $X$, seja de posto coluna completo, tem-se então que na obtencão explí cita do BLUE e do BLUP, considerandol 3.411 e [3.42] tem-se 


$$
V^{-1}=\frac{1}{\sigma_{\theta}^{2}} I_{a b}-\frac{\sigma_{\beta}^{2}}{\sigma_{\theta}^{2}\left(\sigma_{\theta}^{2}+a \sigma_{\beta}^{2}\right)} J_{a} \otimes I_{b}
$$

considerando

$$
X=D_{a}\left(1_{b}\right), \quad \text { obtem-se }
$$

$$
\left(X^{\prime} V^{-1} X\right)=\left[D_{a}(b)-\frac{b \sigma_{\beta}^{2}}{\sigma_{e}^{2}+a \sigma_{\beta}^{2}} J_{a}\right]
$$

e aplicando-se os resultados apresentados em HENDERSON \& SEARLE (1981) e SEARLE (1982) na inversão de [3.53], isto é:

$$
\left(A+b u V^{\prime}\right)^{-1}=A^{-1}-\frac{b}{1+b V^{\prime} A^{-1} u} A^{-1} u V^{\prime} A^{-1}
$$

obtém-se que

$$
\left(X^{\prime} V^{-1} X\right)^{-1}=\left[D_{a}^{-1}(b)+\frac{t}{1+t 1 \cdot D^{-1}(b) 1} D_{a}^{-1}(b) 11 \cdot D_{a}^{-1}(b)\right]
$$

onde

$$
t=\frac{b \sigma_{\beta}^{2}}{\sigma_{e}^{2}+a \sigma_{\beta}^{2}}
$$

e de [3.11] obtém-se que o

$$
\operatorname{BLUE}\left(\alpha_{i}\right)=\bar{y}_{i}
$$

que é obtido também das equacões nomais:

$$
X^{\prime} X a=X^{\prime} y
$$

Assim, observa-se que

$$
\operatorname{var}\left(\operatorname{BLUE}\left(\alpha_{i}\right)\right)=\operatorname{var}\left(\bar{y}_{i}\right)=\frac{1}{b}\left(\sigma_{e}^{2}+\sigma_{\beta}^{2}\right)
$$

e como em [3.12] foi obtida a expressão geral da variância do BLUE, verifica-se que 
$\operatorname{var}(\operatorname{BLU}(\alpha))=-\frac{1}{b}\left[\begin{array}{ccc}\sigma^{2}+\sigma^{2} & \sigma^{2} & \sigma_{\beta}^{2} \\ \sigma_{\beta}^{2} & \sigma^{2}+\sigma_{\beta}^{2} & \sigma^{2} \\ \vdots & \vdots & \vdots \\ \sigma_{\beta}^{2} & \sigma_{\beta}^{2} & \sigma^{2}+\sigma_{\beta}^{2}\end{array}\right]$

$[3.56]$

resultado este, mito conhecido nas áreas da estatística experimental e dos componentes de variancia.

Agora por 13.181 encontrame que

$$
\operatorname{BLUP}(\beta)=\frac{1}{c^{2}+2 c^{2}}\left(\bar{y}_{3},-y^{-}\right) .
$$

Ao supor que em 13.521 a estrutura dos dados $b$ no balancedate, isto $6, k=1,2, \ldots, n_{i}$ e sem considerar-se o termo constante (ou ao fazer una reparametrizasäo na natriz assookad aos efeitos fixos), ten-se que a natriz inversa dos conponentes de varjancia é semelnarte cruzados, assim considerando $x=D\left(1_{r_{i}}\right)$, e substituindo-se en

[3.11] tem-se que

$\left(X^{\prime} V^{-1} X\right)^{-1}=$

$=\left[D\left(n_{2}\right)-\sum_{j=1}^{b} \frac{\sigma_{\beta}^{2}}{\sigma_{e}^{2}+n \cdot \alpha_{\beta}^{2}}\left(n_{1 j}, \ldots, n_{c i j}\right)\left(n_{1 j}, \ldots, n_{a j}\right)\right]$

onde $n_{2}=\sum_{j} n_{i j}$ e $n_{\cdot j}=\sum_{i} n_{i j}$

e

$$
X \cdot V^{-1} y=\left[y_{i}-\sum_{j=1}^{b} \frac{\sigma^{2} n_{i j} y-j \cdot}{\sigma^{2}+n+\sigma^{2}}\right]
$$




$$
\operatorname{BLUE}(\alpha)=\left(X^{\prime} V^{-1} X\right)^{-1} X^{\prime} V^{-1} y x^{\prime}\left(X^{\prime} X\right)^{-1} X^{\prime} y
$$

obtido de [3.58] e [3.59], note que também neste caso a estimativa de a no modelo superparanetrizado é equivalente aquela obtida no modelo de médias, conforme IEMMA(1991).

observacão: É importante considerar que no caso de estruturas de dados balanceados, se a matriz associada aos efeitos fixos for de posto coluna completo, entăo o Blue näo dependerá dos componentes aleatórios, como pode ser observado, por exemplo em [3.51] e [3.55], que como já foi dito é uma consequência de [3.16]. Esta é porém una afirmacão válida en toda estrutura de dados que satisfazem a essas características. 


\section{ESTIMACÃO DOS COMPONENTES DE VARI ÂNCIA}

\subsection{Introducão}

Dada a grande importância dos compenentes de variância no estudo dos BLUE e do BLUP, é conveniente que sejam apresentados alguns mótodos para obtensäo de suas estimativas, embora existan miitos procedimentos. Neste trabalho, são estudados somente aqueles baseados na teoria da distribuicão normal. Ademais säo considerados procedimentos especí ficos no caso de estruturas de dados balanceados e não balanceados.

\section{2. Método da Máxima Verossimilhanca}

Seja o modelo [3.1] o qual pode ser escrito mais explicitamente na forma

$$
y=X \beta+Z_{1} u_{1}+\ldots+Z_{s} u_{s}+e
$$

onde é imposta a restricão de normalidade, ou seja

$$
\begin{gathered}
e n N\left(0, \alpha^{2} I\right) \quad u_{i} \cap N\left(0, \alpha_{i}^{2}\right) \\
i=1, \ldots=
\end{gathered}
$$

$u_{L}$ : é o vetor dos efeitos aleatórios do i-ésimo fator 


$$
y \cap N(X \beta, V)
$$

onde a matriz de variâncias e covariâncias $V$ pode ser reescrita na forma.

$$
V=\sigma^{2} I+\sigma_{1}^{2} Z_{1} Z_{s}^{\prime}+\ldots+c_{s}^{2} Z_{s} Z_{s}^{\prime}
$$

ou também

$$
\begin{aligned}
\frac{V}{\sigma^{2}} & =\left[I+\frac{\sigma^{2}}{\sigma^{2}} Z_{1} Z_{1}^{\prime}+\ldots+\frac{\sigma_{s}^{2}}{\sigma^{2}} Z_{s} Z_{s}^{\prime}\right] \\
& =\left[I+\gamma_{1} Z_{1} Z_{1}^{\prime}+\ldots+\gamma_{s} Z_{s} Z_{s}^{\prime}\right]
\end{aligned}
$$

onde

$$
\begin{gathered}
\gamma_{i}=\sigma_{i}^{2} / \sigma^{2} \\
i=1,2, \ldots, \sigma
\end{gathered}
$$

Pelo fato dá normalidade, então a funcão de verossimilhanca é neste caso:

$L\left(y, X i, \sigma_{i}^{2}\right)=$

$=\left(2 \pi \sigma^{2}\right)^{-\frac{n}{2}}|V|^{-1 / 2} \operatorname{Exp}\left[-\frac{1}{2 \sigma^{2}}(y-X / \beta) \cdot V^{-1}(y-X / 3)\right]$

e o logaritmo da funcão de verossimilhanca fica

$$
\begin{aligned}
\lambda & =-\frac{n}{2} x_{n}(2 \pi)-\frac{n}{2} x_{n} \sigma^{2}-\frac{1}{2} x_{n}|V|- \\
& -\frac{1}{2 \sigma^{2}}(y-X / 3) \cdot V^{-1}(y-X / 3)
\end{aligned}
$$

e então

$$
\frac{\partial \lambda}{\partial / 3}=-\frac{1}{2 \sigma^{2}}\left[-2 X^{\prime} V^{-1} Y+2\left(X^{\prime} V^{-1} X\right) \beta\right]=0
$$




$$
\frac{\partial \lambda}{\partial \sigma^{2}}=-\frac{n}{2 \sigma^{2}}+\frac{1}{2 \sigma^{4}}(y-X \beta)^{\prime} V^{-1}(y-X / \beta)=0
$$

e

$\frac{\partial \lambda}{\partial \gamma_{i}}=-\frac{1}{2} \cdot \frac{\partial}{\partial \gamma_{i}} \operatorname{Ln}|V|-\frac{1}{2 \sigma^{2}}(y-X / \beta)^{\prime}-\frac{\partial V^{-1}}{\partial \gamma_{i}}(y-X / 3) \quad[4.3]$

mas note-se que

$$
\frac{\partial}{\partial \gamma_{i}} \operatorname{Ln}|V|=\frac{1}{|V|} \frac{\partial|V|}{\partial \gamma_{i}}
$$

e Ienbrando que

i) $|V|$ é o determinante da natria V

ii) $V^{-1}=\frac{A d j(V)}{|V|}=\frac{1}{|V|} \frac{\partial|V|}{\partial V}$, onde $A d j(V)$ e a matriz adjunta de $V$, ao substituir en $[4.9]$ tem-se

$$
\begin{aligned}
\frac{\partial}{\partial \gamma_{i}} \sum_{n}|\mathrm{~V}| & =\frac{1}{|V|} \Sigma \Sigma \frac{\partial|V|}{\partial V} \cdot \frac{\partial V}{\partial \gamma_{i}} \\
& =\Sigma \Sigma\left[\frac{1}{|V|} \frac{\partial|V|}{\partial V}\right] \frac{\partial V}{\partial \gamma_{i}} \\
& =\sum_{i} \sum V_{j}^{-1} \frac{\partial V}{\partial \gamma_{i}} ; \text { veja-Se SEARLE } 1982
\end{aligned}
$$

Por outro lado, da definicão do traco de uma matriz nota-se que

$$
\frac{\partial}{\partial \gamma_{i}} \mathscr{L}|V|=\operatorname{Tr}\left[V^{-1} \frac{\partial V}{\partial \gamma_{i}}\right]
$$

e assim ao substituir en [4.8] tem-se

$$
\frac{\partial V}{\partial \gamma_{i}}=-\frac{1}{2} \operatorname{Tr}\left[\mathrm{V}^{-1} \frac{\partial V}{\partial \gamma_{i}^{\prime}}\right]-\frac{1}{2 \sigma^{2}}\left(\mathrm{y}-\mathrm{X}_{\hat{\beta}}\right) \cdot \frac{\partial \mathrm{V}^{-1}}{\partial \gamma_{i}}(\mathrm{y}-\mathrm{X} / \hat{\beta})
$$


De [4.3] tem-se que:

$$
\frac{\partial V}{\partial \gamma_{i}}=Z_{i} Z_{i}^{\prime} \quad \text { e } \frac{\partial V^{-1}}{\partial \gamma_{i}}=-V^{-1} \frac{\partial V}{\partial \gamma_{i}^{\prime}} V^{-1}
$$

portanto

$\frac{\partial V}{\partial \gamma_{i}}=-\frac{1}{2} \operatorname{Tr}\left(V^{-1} Z_{i} Z_{i}^{\prime}\right)+\frac{1}{2 \sigma^{2}}(y-X \beta)^{\prime} V^{-1} Z_{i} Z_{i}^{\prime} V^{-1}(y-X \beta)$

De $[4.6]$ e $[4.7]$ pode-se observar que tanto $\beta$ como $\sigma^{2}$ dependem linearmente de $\gamma^{-1}$, ou seja

$$
\begin{gathered}
\hat{\beta}\left(\gamma_{i}\right)=\left(X^{\prime} V^{-1} X\right)^{-1} X^{\prime} V^{-1} y \\
n \tilde{\sigma}^{2}\left(\gamma_{i}\right)=y^{\prime} V^{-1} y-y^{\prime} V^{-1} X\left(X^{\prime} V^{-1} X\right)^{-1} X^{\prime} V^{-1} y
\end{gathered}
$$

A solucão de [4.11] não é imediata, é necessário o uso de métodos numéricos na sia obtencão

Una forma de se contornar o problema na estimação, en [4.11], é reescrevendo $V$ de $\left[3.41\right.$ ] em funcão dos $\lambda_{\mathbf{P}}$. Pelo fato de que $\lambda_{\mathbf{P}}=\mathrm{T}_{\mathbf{P}} \Theta_{\mathbf{P}}$ e, como $\mathrm{T}_{\mathbf{P}}$ é não singular então

$$
V=\Delta T_{P}^{-1} \lambda_{P}
$$

dessa forma a matriz dos componentes de variância será obtida pela soma dos componentes ortogonais, com o qual facilita-se a estimatição dos componentes. Esta é a forma de obtenção de V sugerida por HOCKING (1985). Neste trabalho apresenta-se uma forma mais simples na obtencão dessas partiç̃es ortogonais com o gual facilita-se a sua estimasão.

Para esclarecer esta forma de $V$, seja o modelo apresentado em [3.43], onde por [3.41] vimos que 
$V=\sigma_{e}^{2} I_{I J K}+\sigma_{\gamma}^{2} I_{X J} \otimes J_{K}+\sigma_{\alpha}^{2} I_{I} \otimes J_{J K}+\sigma_{\beta}^{2} J_{I} \otimes I_{J} \otimes J_{K}$,

e de [3.44] tem-se que

$$
\begin{gathered}
\lambda_{0}=\sigma_{e}^{2} \Rightarrow \sigma_{e}^{2}=\lambda_{0} \\
\lambda_{\alpha \beta}=\sigma_{e}^{2}+K \sigma_{\gamma}^{2} \Rightarrow \sigma_{\gamma}^{2}=\frac{1}{K}\left[\lambda_{\alpha \beta}-\lambda_{0}\right] \\
\lambda_{\alpha}=\sigma_{e}^{2}+K \sigma_{\gamma}^{2}+J K \sigma_{\alpha}^{2} \Rightarrow \sigma_{\alpha}^{2}=\frac{1}{J K}\left[\lambda_{\alpha}-\lambda_{\alpha \beta}\right] \\
\lambda_{\beta}=\sigma_{e}^{2}+K \sigma_{\gamma}^{2}+I K \sigma_{\beta}^{2} \Rightarrow \sigma_{\beta}^{2}=\frac{1}{I K}\left[\lambda_{\beta}-\lambda_{\alpha \beta}\right] \\
\lambda_{\mu}=\sigma_{e}^{2}+K \sigma_{\gamma}^{2}+J K \sigma_{\alpha}^{2}+I K \sigma_{\beta}^{2}=\left[\lambda_{\alpha}+\lambda_{\beta}-\lambda_{\alpha \beta}\right]
\end{gathered}
$$

ou ainda: considerando:

$$
\left[\begin{array}{l}
\lambda_{0} \\
\lambda_{\alpha \beta} \\
\lambda_{\alpha} \\
\lambda_{\beta} \\
\lambda_{u}
\end{array}\right]=\lambda_{P}=\left[\begin{array}{llll}
1 & 0 & 0 & 0 \\
1 & K & 0 & 0 \\
1 & K & J K & 0 \\
1 & K & 0 & I K \\
1 & K & J K & I K
\end{array}\right]\left[\begin{array}{c}
\sigma_{e}^{2} \\
\sigma_{\gamma}^{2} \\
\gamma \\
\sigma_{\alpha}^{2} \\
\sigma_{\beta}^{2}
\end{array}\right]
$$

e fazendo-se

$$
\dot{T}_{P}=\left[\begin{array}{llll}
1 & 0 & 0 & 0 \\
1 & K & 0 & 0 \\
1 & K & J K & 0 \\
1 & K & 0 & I K
\end{array}\right]
$$

De onde $e=\dot{T}_{\mathbf{P}}^{-1} \hat{\lambda}_{P} ;$ onde $I_{I J K}=I_{N} ; J_{I J K}=J_{N}$

Observa-se de $[4.14]$

$V=\lambda_{0} I_{N}+\frac{1}{J K} \lambda_{\alpha} I_{I} \otimes J_{J K}+\frac{1}{I K} \lambda_{\beta} J_{I} \otimes I_{J} \otimes J_{K}+$ 


$$
\begin{aligned}
& +\frac{1}{K} \lambda_{\alpha \beta}\left[I_{I J} \otimes J_{K}-\frac{1}{J} I_{I} \otimes J_{J K}-\frac{1}{I} J_{I} \otimes I_{J} \otimes J_{K}\right]- \\
& -\frac{1}{K} \lambda_{O} I_{I J} \otimes J_{K}
\end{aligned}
$$

Adicionando-se à expressão anterior as parcelas:

$$
\pm \lambda_{a} \frac{1}{N} J_{N} ; \lambda_{\beta} \frac{1}{N} J_{N} ; \lambda_{\alpha \beta} \frac{1}{N} J_{N}
$$

obtém-se que

$$
\begin{aligned}
& V=\lambda_{0}\left[I_{N}-\frac{1}{K} I_{X J} \otimes J_{K}\right]+\lambda_{\alpha}[\underbrace{\left[\frac{1}{J K} I_{I} \otimes J_{K K}-\frac{1}{N} J_{N}\right]}_{A_{0}}+ \\
& +\lambda_{\beta} \underbrace{\left[\frac{1}{I K} J_{I} \otimes I_{J} \otimes J_{K}-\frac{1}{N} J_{N}\right]}_{A_{2}}
\end{aligned}
$$

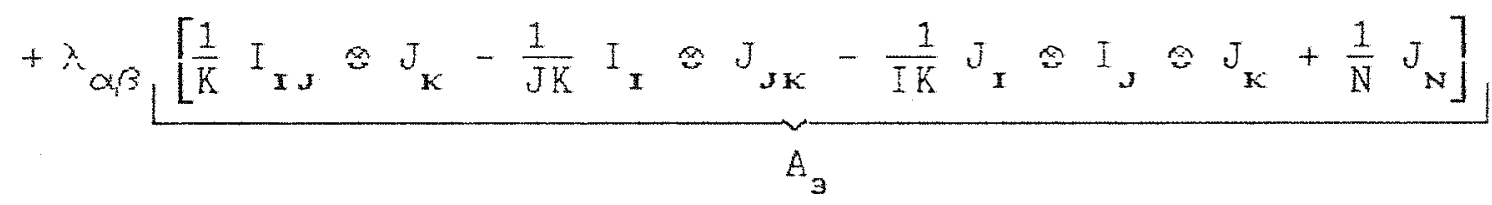

$$
\begin{aligned}
& +\left(\lambda_{\alpha}+\lambda_{\beta}-\lambda_{\alpha \beta}\right) \underbrace{\frac{1}{N} J_{N}}_{A_{H}}
\end{aligned}
$$$$
V=\lambda_{0} A_{0}+\lambda_{\alpha} A_{1}+\lambda_{\beta} A_{2}+\lambda_{\alpha \beta} A_{3}+\lambda_{\mu} A_{\mu}
$$

\section{Safisfazendo às seguintes condicões}

i) $\quad A_{t} A_{t}=\left[\begin{array}{l}0 \text { se } t \neq t^{\prime} \\ A_{t} \text { se } t=t^{\prime}\end{array}, t^{\prime}=0,1,2,3\right.$

e além disso $A_{t} A_{\mu}=0, \forall_{t}$ 
ii) $A_{t}=A_{t}^{\prime}$

iii) $A_{t}=A_{t}^{-1}$ ou seja são matrizes ortogonais e desse modo a matriz inversa em $\{4.16\}$ é imediata, ou seja

$$
V^{-1}=\frac{1}{\lambda_{0}} A_{0}+\frac{1}{\lambda_{\alpha}} A_{1}+\frac{1}{\lambda_{\beta}} A_{2}+\frac{1}{\lambda_{\alpha \beta}} A_{9}+\frac{1}{\lambda_{\mu}} A_{\mu}
$$

Observacão:

Como

$$
\begin{aligned}
& y_{i, j k}=\left[y_{i j k}-\frac{1}{K} y_{i j}\right]+\left(\frac{1}{J K} y_{i} \ldots-\frac{1}{N} y_{\ldots}\right] \\
& +\left[\frac{1}{I K} y_{. j}-\frac{1}{N} y_{\ldots}\right]+\left(\frac{1}{K^{2}} y_{i,}-\frac{1}{J K} y_{i}-\frac{1}{I K} y_{. j}+\frac{1}{N} y_{\ldots}\right) \\
& +\frac{1}{N} y \ldots
\end{aligned}
$$

ou seja $\mathrm{y}_{2 j k}$ é reescrito nos termos das particães ortogonais do modelo, então é fácil estabelecer una relaçäo direta entre estas particões ortogonais e as matrizes $A_{t}$ e $A_{\mu}$ em [4.16]. Note-se que a relacão é imediata se são feitos os produtos de Kronecker segundo as seguintes variáveis indicadoras.

$$
\delta=\left\{\begin{array}{l}
\text { I cada vez que um sub-í ndice aparece na resposta } \\
J \text { caso contrário }
\end{array}\right.
$$

assim por exemplo

$$
\begin{gathered}
\frac{1}{J K} y_{i} \ldots-\frac{1}{N} y_{\ldots} \Rightarrow \frac{1}{J K} I_{I} \otimes J_{J} \otimes J_{K}-\frac{1}{N} J_{I} \otimes J_{J} \otimes J_{K} \\
=\frac{1}{J K} I_{I} \otimes J_{J K}-\frac{1}{N} J_{N}=A_{1}
\end{gathered}
$$


com esta observaça, pode-se estabelecer de forma simples a estrutura de V dada en [4.16].

A grande utilidade de escrever $V$ na forma de [4.16] consiste no fato de que a funcão de verossimilhanca $[4.4\}$ é amplamente simplificada, ela fica

$$
\begin{aligned}
& L\left(y, \mu, \sigma^{2}\right)= \\
& \quad=\left(2 \pi \sigma^{2}\right)^{-n / 2}|V|^{-1 / 2} \operatorname{Exp}\left(\left[-\frac{1}{2}-\right]\left[\frac{N(\bar{y}-\mu)^{2}}{\lambda_{\mu}}+\sum_{t=0}^{q} \frac{y^{\prime} A_{t} y}{\lambda_{t}}\right]\right)[4.19]
\end{aligned}
$$

- o logaritmo da funcão é

$$
\sum_{n}(L)=-\frac{N}{2} \operatorname{sen} 2 \pi-\frac{1}{2} \operatorname{sen}|V|-\frac{1}{2}\left[\frac{(\vec{y}-\mu)^{2}}{\lambda \mu}+\sum_{t=0}^{q} \frac{y^{\prime} A t y}{\lambda t}\right][4.20]
$$

de maneira que

$$
\frac{\partial \ln (L)}{\partial \lambda_{t}}=\frac{y^{\prime} \hat{A}_{t} y}{2 \lambda_{t}^{2}}-\frac{1}{2} \operatorname{Tr}^{2}\left[V^{-1} \frac{\partial V}{\partial \lambda_{t}}\right]
$$

mas neste caso

$$
\begin{aligned}
\operatorname{Tr}\left(V^{-1} \frac{\partial V}{\partial \lambda_{t}}\right] & =\operatorname{Tr}\left[\left(\frac{1}{\lambda_{0}} A_{0}+\frac{1}{\lambda_{1}} A_{1}+\ldots+\frac{1}{\lambda_{t}} A_{t}+\ldots \frac{1}{\lambda_{q}} A_{q}\right) A_{t}\right] \\
& =\operatorname{Tr}\left[\frac{1}{\lambda_{t}} A_{t}\right]=\frac{1}{\lambda_{t}} \operatorname{Tr}\left(A_{t}\right)
\end{aligned}
$$

e pelas propriedades de $A_{t}$, então tem-se

$$
\operatorname{Tr}\left(A_{t}\right)=r\left(A_{t}\right)=r_{t}
$$

assim, 


$$
\operatorname{Tr}\left(V^{-1} \frac{\partial V}{\partial \lambda_{t}}\right]=\frac{1}{\lambda_{t}} r_{t}
$$

onde $r_{t}$ é o posto da matriz $A_{t}$.

De [4.21] igualando a zero, obtém-se

$$
y^{\prime} A_{t} y=\lambda_{t} r_{t} \Rightarrow \hat{\lambda}_{t}=\frac{y^{\prime} A_{t} y}{r_{t}}
$$

a fazer que $Q_{t}=y^{*} A_{t} y$ então

$$
\hat{\lambda}_{t}=Q_{t} / r_{t}
$$

pela invariância dos estimadores de náxima verossimilhano, as estimativas los componentes de variância săo obtidos.

Assim continuando com o exemplo

$$
\begin{aligned}
\hat{\alpha}_{e}^{2}=\hat{\lambda}_{0} & =\frac{y^{\prime} A_{0} y}{r_{0}} ; r_{0}=\operatorname{rank}\left(A_{0}\right) \\
\hat{\sigma}_{\gamma}^{2} & =\frac{1}{K}\left[\hat{\lambda}_{\alpha \beta}-\hat{\lambda}_{0}\right] \\
\hat{\sigma}_{\alpha}^{2} & =\frac{1}{J K}\left[\hat{\lambda}_{\alpha}-\hat{\lambda}_{\alpha \beta}\right] \\
\hat{\sigma}_{\beta}^{2} & =\frac{1}{I K}\left[\hat{\lambda}_{\beta}-\hat{\lambda}_{\alpha \beta}\right]
\end{aligned}
$$

Das propriedades de distribuicöes de formas quadráticas demonstra-se que

$$
\frac{Q_{t}}{\lambda_{t}} \cap x^{2}\left(r_{t}\right) \Leftrightarrow Q_{t} \cap \lambda_{t} \chi^{2}\left(r_{t}\right)
$$

E então

$$
\operatorname{Var}\left(Q_{t}\right)=\lambda_{t}^{2} \operatorname{Var}\left(\lambda^{2}\left(r_{t}\right)\right)
$$




$$
\begin{gathered}
=2 \lambda_{t}^{2} r_{t} . \\
\operatorname{Var}\left(Q_{t} / r_{t}\right)=2 \lambda_{t}^{2} / r_{t} .
\end{gathered}
$$

ou seja

$$
\operatorname{Var}\left(\hat{\lambda}_{t}\right)=2 \lambda_{t}^{2} / r_{t}
$$

e como

$$
\hat{\lambda}_{P}=\dot{T}_{P} \hat{\theta}_{P} \Rightarrow \hat{\theta}_{P}=\dot{T}^{-1} \hat{\lambda}_{P}
$$

então

$$
\operatorname{Var}\left(\hat{\Theta}_{P}\right)=\dot{T}_{P}^{-1} \operatorname{Diag}\left(2 \lambda_{t}^{2} / \gamma_{t}\right) \dot{T}_{P}^{-1}
$$

desse modo:

$$
\begin{gathered}
E\left[\hat{\lambda_{t}}\right]=E\left[Q_{t} / r_{t}\right] \\
=\frac{1}{r_{t}} E\left[Q_{t}\right]=\frac{1}{r_{t}}\left[\operatorname{Tr}\left(A_{t} V\right)+\mu^{\prime} A_{t} \mu\right] \\
=\frac{1}{r_{t}}\left[\left(\operatorname{VEC} A_{t}^{\prime}\right)^{*} \operatorname{VECV}+\mu^{\prime} A_{t} \mu\right]=\lambda_{t}
\end{gathered}
$$

onde VEC é um operador definido de tal forma que se

$$
x=\left[\begin{array}{ll}
a & b \\
c & d
\end{array}\right] \Rightarrow \operatorname{VEC} x=\left[\begin{array}{l}
a \\
b \\
c \\
d
\end{array}\right]
$$

- qual pode ser amplamente estudado en HENDERSON \& SEARLE (1979), entre outros.

Se todos os quadrados médios obtidos a partir de [4.22], são escritos na forma linear, tem-se 


$$
S \hat{\lambda}_{p}=S_{0} \hat{\lambda}_{0}+S_{1} \hat{\lambda}_{1}+\ldots+S_{q} \hat{\lambda}_{q}+S_{L_{H}} \hat{\lambda}_{\mu},
$$

então pelo fato de que $E\left(\hat{\lambda}_{t}\right)=\lambda_{t}$, ou seja estimador ser não viesado

i) $\operatorname{Var}\left(\operatorname{Var}\left(\hat{\lambda}_{t}\right)\right)=\frac{2\left[y^{\prime} A_{t} y\right]^{2}}{r_{t}^{2}\left(r_{t}+2\right)}=\frac{2 \lambda^{2}}{\left(r_{t}+2\right)}$

ten-se que na sua estrutura geral

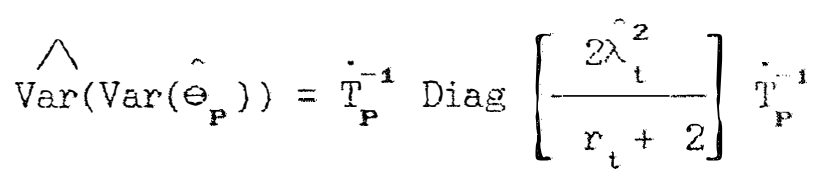

nota-se também que

ii) $\hat{E}\left[\operatorname{Var}\left(\operatorname{Var}\left(\hat{\lambda}_{t}\right)\right]=E\left[\frac{2 \hat{\lambda}^{2}}{r_{t}+2}\right]=\frac{2}{r_{t}+2}\left(\operatorname{Var}\left(\hat{\lambda}_{t}\right)+\left(E\left(\hat{\lambda}_{t}\right)\right)^{2}\right]\right.$

$$
=\frac{2 \lambda_{t}^{2}}{r_{t}}
$$

Este resultado é estendido na sua forma feral ou seja

$$
E\left[\operatorname{Diag}\left(\frac{2 \lambda_{t}^{2}}{r_{t}+2}\right)\right]=\operatorname{Diag}\left[2 \frac{\lambda^{2}}{r_{1}}\right]
$$

Dos resultados i) e ii) verifica-se un

$$
E\left[\hat{\lambda}_{\mathbf{p}} \hat{\lambda}_{p}^{\prime}-\operatorname{Diag}\left[2 \frac{\lambda_{t}^{2}}{r_{t}}\right]\right]=\lambda_{y} \cdot \lambda_{y}
$$

No contexto do modelo $[3.43]$, Jivmh, in un consideracão que 


$$
\dot{\mathrm{T}}_{\mathbf{P}}^{-1}=\left[\begin{array}{ccll}
1 & 0 & 0 & 0 \\
-1 / \mathrm{K} & 1 / \mathrm{K} & 0 & 0 \\
0 & -1 / \mathrm{JK} & 1 / \mathrm{JK} & 0 \\
0 & -1 / \mathrm{IK} & 0 & 1 / \mathrm{IK}
\end{array}\right]
$$

obtem-se que

$\widehat{\operatorname{Var}}\left(\operatorname{Var}\left(\hat{\theta}_{\mathbf{P}}\right)\right)=\dot{\mathrm{T}}_{\mathbf{P}}^{-1} \operatorname{Diag}\left[\frac{2 \hat{\lambda}_{t}^{2}}{r_{t}+2}\right] \dot{T}_{\mathbf{P}}^{-1}=$

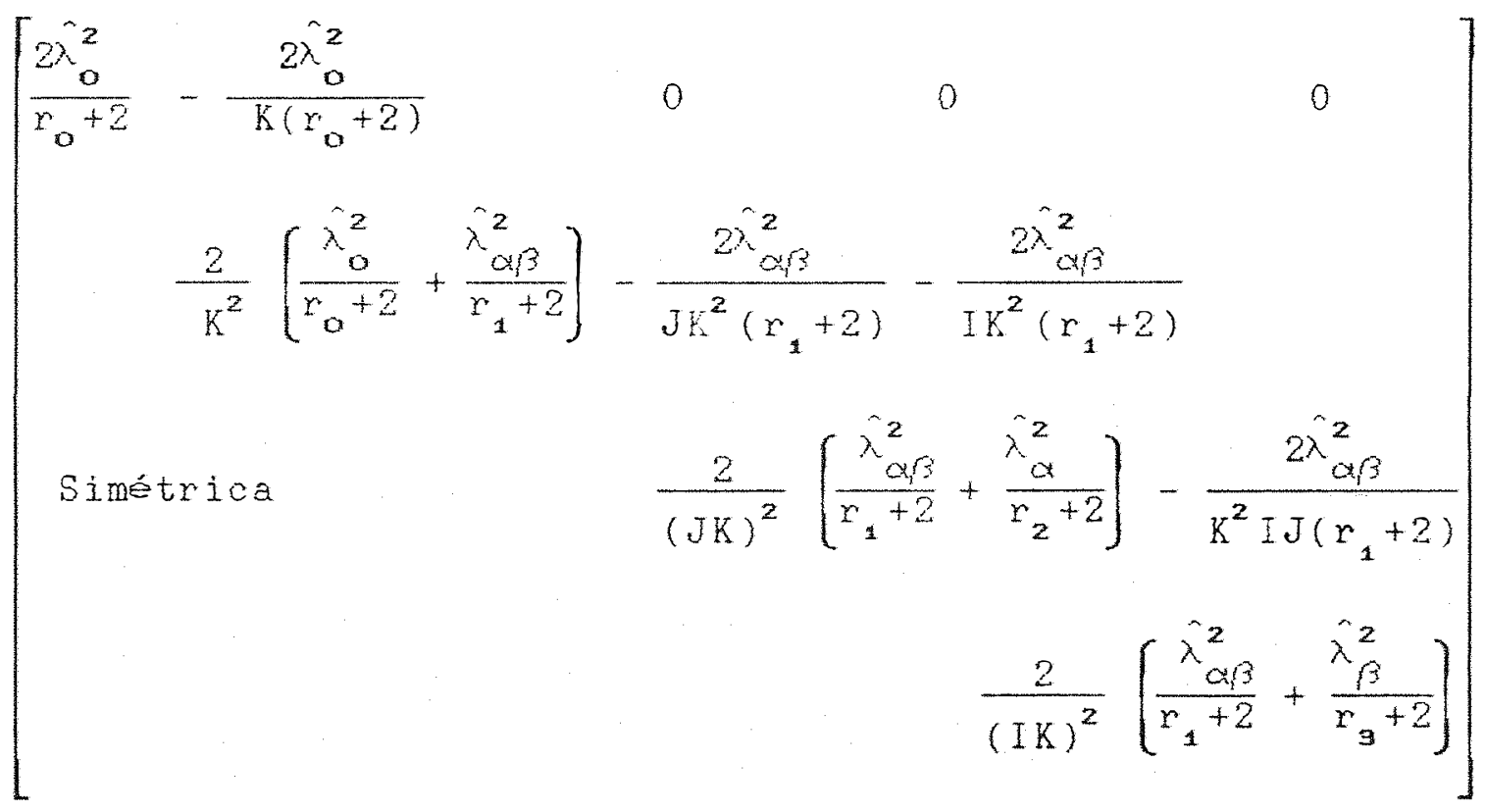

onde $r_{0}, r_{1}, r_{2}$ e $r_{3}$ são os graus de liberdade associados efeitos e são equivalentes aos postos das natrizes associadas às partič̃es ortogonais do modelo.

Outra vantagem existente em se escrever a matriz de variâncias e covariâncias da forma [4.16], está no fato de que além de obter-se as estimativas em [4.22], dada a suposicão de normalidade pode-se obter o r-ésimo monento, usando-se o resultado apresentado por SEARLE (1971) do r-ésino cumulante. 
Chega-se em

$$
\begin{aligned}
\operatorname{Var}\left[y^{\prime}\left(A_{t}+A_{t},\right) y\right] & =\operatorname{Var}\left[y^{\prime} A_{t} y\right]+\operatorname{Var}\left(y^{\prime} A_{t}, y\right) \\
& -2 \operatorname{Cov}\left[y^{\prime} A_{t} y ; y^{\prime} A_{t}, y\right]
\end{aligned}
$$

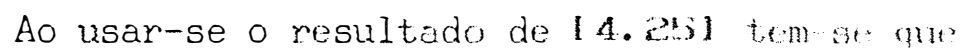

$\operatorname{Cov}\left(y^{\prime} A_{t} y ; y^{\prime} A_{t}, y\right)=2 \operatorname{Tr}\left(A_{t} V A_{t} V\right)+4 H^{\prime} A_{t} V A_{t} \cdot H$

mas pelo fato de que

$\operatorname{Tr}\left(A Z^{\prime} B Z(C)=(V E C Z)^{\prime}\left(A^{\prime} C^{\prime} \circ B\right)\right.$ VEC Z

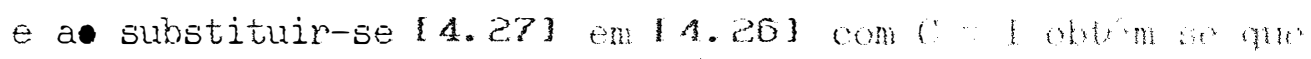

$\operatorname{Cov}\left(y^{\prime} A_{t} y ; y^{\prime} A_{t} . y\right)=2(\operatorname{VEC} V)^{\prime}\left(A_{t} \circ A_{t}\right) \operatorname{VBC} V$

$$
+4, U^{\prime} A_{t} V A_{t}, H
$$

e pelo fato de que

$$
A_{t} V=A_{t}\left[\lambda_{0} A_{0}+\lambda_{1} A_{1}+\ldots+\lambda_{1} A_{1}+\lambda_{u} \Lambda_{u}\right]=\lambda_{1} A_{1}
$$

tem-se finalmente que

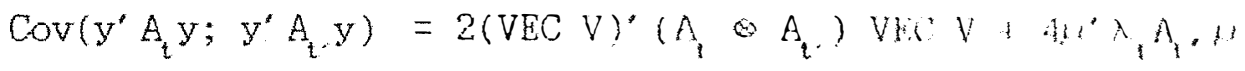
no entanto $E\left(y^{*} A_{t} y ; y^{*} A_{t} y\right)=\left(\operatorname{Tr} A_{t} V\right)\left(\operatorname{Tr} A_{t} V\right)+2 \operatorname{Tr} A_{t} V A_{t} \cdot V$

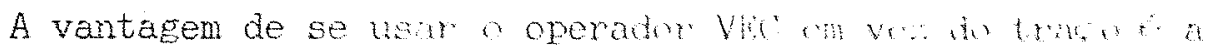
facilidade resultante nos cilmules compun inmin.

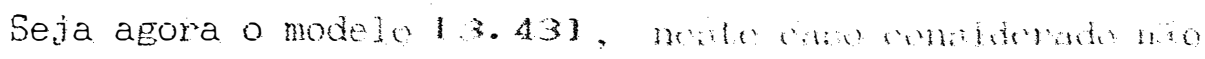


balanceado, ou seja $k=1,2, \ldots, n_{k j}$, entro a decomposicão de [4.18] fica:

$$
\begin{aligned}
& y_{i j k}=\left(y_{i, k}-\frac{1}{n_{i j}} y_{i j}\right)+\left(\frac{1}{n_{i}} y_{i}-\frac{1}{N} \cdot \ldots\right)+ \\
& +\left[\frac{1}{n_{. j}} y_{. j}-\frac{1}{N} y \ldots\right]+\left(-\frac{1}{n_{i j}} y_{1}-\frac{1}{n_{i}} y_{2} \ldots-\right. \\
& \left.-\frac{1}{n_{i . j}} y_{. j .}+\frac{1}{N} y \ldots\right)+\frac{1}{N} y \ldots
\end{aligned}
$$

onde

$$
\begin{aligned}
& n_{i}=\sum_{j} n_{i j} \quad n_{j}=\sum_{i} n_{1} \\
& N=\sum_{i} n_{i}=\sum_{j} n_{j}=\sum_{i} n_{1}
\end{aligned}
$$

Neste caso pela mesma relacão ammintinla na obervacào [4.1], nota-se por exemplo, wue $A_{1} e ́$ obtidn mrindo-se do fato

$$
\frac{1}{n_{i}} y_{i} . .-\frac{1}{N} y_{\ldots}
$$

tem-se que

$$
A_{1}=\underbrace{1}_{i=1} \frac{1}{n_{i}} J_{n_{i}}-\frac{1}{N} \cdot{ }_{N}
$$

ou explicitamente 
$A_{1}=\left[\begin{array}{cccc}\frac{1}{n_{1}} J_{n_{1}} & \phi & \phi \\ & \frac{1}{n_{2}} J_{n_{2}} & \phi \\ & & \vdots \\ & & \frac{1}{n_{I}} J_{n_{I}}\end{array}\right]-\frac{1}{N}\left[\begin{array}{cccc}1 & 1 & \ldots & 1 \\ 1 & 1 & \ldots & 1 \\ \vdots & \vdots & & \vdots \\ 1 & 1 & \ldots & 1\end{array}\right]_{N}$

onde

$$
J_{n_{i}}=n_{n_{i}}\left[\begin{array}{cccc}
1 & 1 & \cdots & 1 \\
\vdots & \vdots & & \vdots \\
1 & 1 & & 1
\end{array}\right]_{n_{2}}
$$

escrevendo-se assim $V$ na forma expressa em [4.16], cono neste caso a estrutura de dados é não balanceado tem-se

$$
E\left(y^{\prime} A_{0} y\right)=E\left(Q_{0}\right)=(N-S) \sigma_{e}^{2}
$$

$$
\begin{aligned}
E\left(y^{\prime} A_{1} y\right) & =E\left(Q_{1}\right)=\left(N-K_{1}\right) \sigma_{\alpha}^{2}+\left(K_{3}-K_{2}\right) \sigma_{\beta}^{2}+ \\
& +\left(K_{3}-K_{23}\right) \sigma_{\alpha \beta}^{2}+(I-1) \sigma_{\theta}^{2}
\end{aligned}
$$

$$
\begin{aligned}
E\left(y^{\prime} A_{2} y\right) & =E\left(Q_{2}\right)=\left(K_{4}-K_{1}\right) \sigma_{\alpha}^{2}+\left(N-K_{2}\right) \sigma_{\beta}^{2}+ \\
& +\left(K_{4}-K_{23}\right) \sigma_{\alpha \beta}^{2}+(J-1) \sigma_{e}^{2}
\end{aligned}
$$$$
E\left(y^{\prime} A_{3} y\right)=E\left(Q_{3}\right)=\left(K_{1}-K_{4}\right) \sigma_{\alpha}^{2}+\left(K_{2}-K_{3}\right) \sigma_{\beta}^{2}+
$$$$
+\left(N+K_{23}-K_{3}-K_{4}\right) \sigma_{\alpha \beta}^{2}+(S-I-J+1) \sigma^{2}
$$

$E\left[y^{\prime} A_{\mu} y\right]-E\left(Q_{\mu}\right)=N \mu^{2}+K_{1} \sigma_{\alpha}^{2}+K_{2} \sigma_{\beta}^{2}+K_{23} \sigma_{\alpha \beta}^{2}+\sigma_{e}^{2}$

onde

$$
\mathrm{K}_{1}=\frac{1}{\mathrm{~N}} \sum_{i=1}^{\mathrm{I}} \mathrm{n}_{i}^{2} ; \quad \mathrm{K}_{2}=\frac{1}{\mathrm{~N}} \sum_{j=1}^{J} \mathrm{n}_{-j}^{2} ;
$$




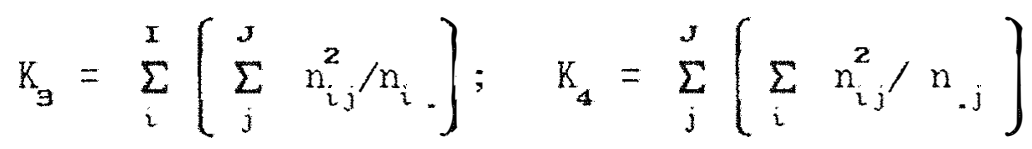

S: número de caselas nã • vazias.

De onde são ebtidas as estimativas das componentes dá variância:

$$
\hat{\sigma}_{e}^{2}=\hat{\lambda}_{0}
$$

onde

$$
\hat{\lambda}_{0}=\frac{y^{\prime} A_{0} y}{r_{0}}=\frac{y^{\prime} A_{0} y}{N-S}
$$

Entretanto as estimativás dos outros componentes são:

$$
\hat{\theta}_{\mathrm{p}}=\left[\begin{array}{c}
\hat{\sigma}_{\alpha}^{2} \\
\hat{\sigma}_{\beta}^{2} \\
\hat{\sigma}_{\beta}^{2} \\
\hat{\sigma}_{\alpha \beta}^{2}
\end{array}\right]=\dot{\mathrm{T}}_{\mathrm{p}}^{-1} \hat{\lambda}_{\mathrm{P}}
$$

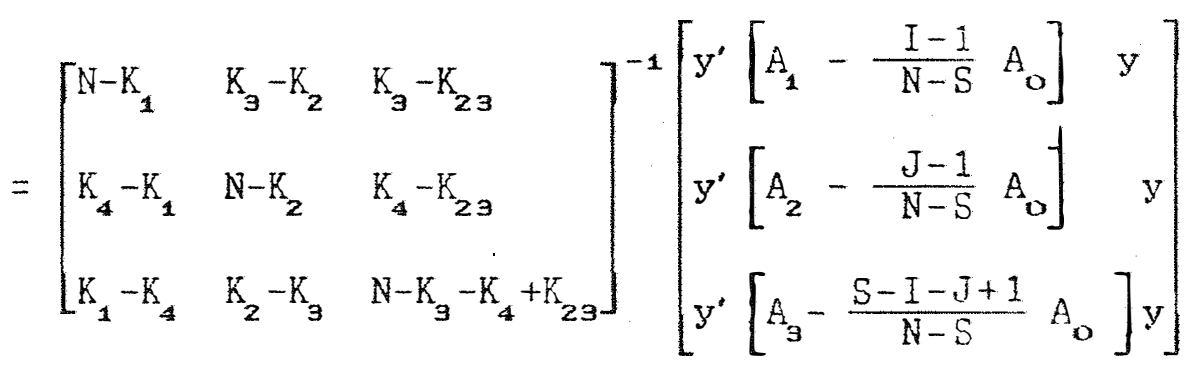

$[4.35]$

4. 3. Cálculo Assintótico dos Componentes da Variancia

No caso de ter-se a estrutura de dados obtidos a partir do temanh da anostra "grande" ou, ainda quando nso for possí vel a 
decomposição linear de $\mathrm{V}$ da forma [4.16], torna-se necessário o uso de processos iterativos na estimacão dos parâmetros de interesse. Muitos procedimentos tèm sido desenvolvidos para se obter estes resultados. Entre os algorí tmos mais conhecidos estão: i) O de Newton-Raphson, que é utilizado na obtencão de pontos de máximo de uma funcão na iteraçà̃o $(i+1)$; i $i)$ o algoritmo a partir dos Scores de Eisher, que esta baseado nas esperancas das primeiras derivadas. Este algorí tmo tem melhor comportamento que o de Newton-Raphson nos pontos mais distantes das estimativas procuradas, no entanto, o algorí tmo de Newton-Raphson tende-se a comportar melhor nas vizinhans as do ponto de máximo; iu) Outro algorítmo usado amplamente nas estimativas de máxima verossimilhano a quando o conjunto de dadosé incompleto ou não observável é o algorí tmo EM, esse algorí tmo consiste basicamente en dois passos ou passo $E$ (esperanoa) e passo $M$ (maximizaça).

Neste trabalho são apresentados apenas os dois primeiros algorítmos, os quais estão baseados nas estimativas de máximá verossimilhanc a.

Seja o logorítmo da funcão de verossimilhanca apresentado em [4.5] onde

$$
\underline{\beta}=\left[\beta_{1}, \ldots, \beta_{k}\right] \text { e } \underline{\sigma}^{2}=\left[\sigma_{1}^{2}, \ldots, \sigma_{s}^{2}, \sigma_{0}^{2}\right]
$$

já foram obtidas na secão 4.2 os seguintes resultados

$$
\frac{\partial \lambda}{\partial \beta}=-\frac{1}{2 \sigma^{2}}\left[-2 X^{\prime} V^{-1} y+2 X^{\prime} V^{-1} X \beta\right]=s_{\beta}
$$

então

$$
\frac{\partial^{2} \lambda}{\partial^{2} \beta}=-\left(X^{\prime} V^{-1} X\right)=\left[H_{\beta \beta}\right]
$$


e o seu valor esperado

$$
E\left[H_{\beta \beta}\right]=-\left(X^{\prime} V^{-1} X\right)
$$

$\operatorname{assim}$

$$
\begin{gathered}
I_{\beta \beta}=-E\left[H_{\beta \beta}\right]=\left(X^{\prime} V^{-1} X\right) \\
\frac{\partial \lambda}{\partial \sigma_{i}^{2}}=-\frac{1}{2} \operatorname{Tr}\left[V^{-1} V^{-1} \frac{\partial V}{\partial \sigma_{i}^{2}}\right]+ \\
+\frac{1}{2} \operatorname{Tr}\left[V^{-1}(y-X \beta)(y-X \beta)^{\prime} V^{-1}-\frac{\partial V}{\partial \sigma_{i}^{2}}\right] \\
=\frac{1}{2} \operatorname{Tr}\left[V^{-1}(y-X \beta)(y-X \beta)^{\prime} V^{-1} \frac{\partial V}{\partial \partial_{i}^{2}}\right]
\end{gathered}
$$

e dos resultados obtidos em SEARLE (1992), JENRICH \& SCHLUCHT (1986) e, HARTLEY \& VAUGHN (1972), segre- se? que

$$
\frac{\partial \lambda}{\partial \sigma_{i}^{2}}=\frac{1}{2} \operatorname{Tr}\left[\mathrm{V}^{-1}(\mathrm{y}-\mathrm{X} \beta)(\mathrm{y}-\mathrm{X} \beta)^{\prime} \mathrm{V}^{-1} \mathrm{Z}_{i} \mathrm{Z}_{i}\right]=S \sigma^{2}
$$

no entanto a segunda derivada e o selu valor esperado ficam

$$
\begin{aligned}
{\left[\mathrm{H}_{\partial \sigma}\right] } & \left.=\frac{\partial^{2} \lambda}{\partial \sigma_{i}^{2} \partial \sigma_{j}^{2}}=\frac{1}{2} \operatorname{Tr}\left[\left[\mathrm{V}^{-1}(\mathrm{y}-\mathrm{X} \beta)(\mathrm{y}) \mathrm{W}\right)^{\prime}-\mathrm{V}\right] \mathrm{V}^{-1} \frac{\partial^{2} \mathrm{~V}}{\partial \sigma_{i}^{2} \partial \sigma_{j}^{2}}\right]- \\
& -\frac{1}{2} \operatorname{Tr}\left[\mathrm{V}^{-1} \frac{\partial \mathrm{V}}{\partial \sigma_{i}^{2}} \mathrm{~V}^{-1} 2(\mathrm{y}-\mathrm{X} / 3)\left(\mathrm{y}-\mathrm{X}_{i}\right)^{\prime} \cdot \mathrm{V}\right] \mathrm{V}^{-1} \frac{\partial \mathrm{V}}{\partial \sigma_{j}^{2}} \quad 14.39
\end{aligned}
$$




$$
\begin{aligned}
E\left[H_{\partial \sigma}\right] & =-\frac{1}{2} \operatorname{Tr} V^{-1} \frac{\partial V}{\partial \sigma_{i}^{2}} V^{-1} \frac{\partial V}{\partial \sigma^{2}} \\
& =-\frac{1}{2} \operatorname{Tr} V^{-1} Z_{i} Z_{i}^{\prime} V^{-1} Z_{j} Z_{j}^{\prime} \\
I_{c y O}= & -E\left[H_{\alpha \sigma}\right]=\frac{1}{2} \operatorname{Tr} V^{-1} Z_{i} Z_{i}^{\prime} V^{-1} Z_{j} Z_{j}^{\prime}
\end{aligned}
$$

agora

$$
\begin{gathered}
{\left[H_{\beta O}\right]=\frac{\partial^{2} \lambda}{\partial \beta_{j} \partial \sigma_{i}^{2}}=X^{\prime} V^{-1} \frac{\partial V}{\partial \sigma_{i}^{2}} V^{-1}(y \cdots X)} \\
=-X \frac{\partial V^{-1}}{\partial \nu_{j}^{2}}(y-X) \\
E\left[H_{\beta C}\right]=0,
\end{gathered}
$$

observa-se, então que a matriz Hessiana.

$$
\mathrm{H}=\left[\begin{array}{ll}
H_{\gamma \beta} & H_{\beta \gamma} \\
H_{\gamma \beta} & H_{\alpha}
\end{array}\right]
$$

é no caso em questão, positiva definida.

Expandindo-a a seguir, através da serte de Taylor a funcão Logverossimilhanca $\lambda(y, \beta, \sigma)=\lambda(y, \delta)=$

$$
\begin{aligned}
& =\lambda\left(\delta^{(i)}\right)+s\left(\delta^{(1)}\right) \cdot\left(\delta-\delta^{(1)}\right)+ \\
& +\frac{1}{2}\left(\delta-\delta^{(i)}\right) \cdot 11\left(\delta^{(1)}\right)(\delta \\
& +0\left[1\left|s-\delta^{(1)}\right| 1\right]
\end{aligned}
$$


onde $\delta=\left[\begin{array}{l}\beta \\ \gamma\end{array}\right]$ e $\bullet$ é á ordem de convergencia da forma $a_{n}=0\left(r_{n}\right)$ Be

$$
\lim _{n \rightarrow \infty} \frac{a_{n}}{g_{n}}=0\left(a_{n} \Leftrightarrow 0 \text { "Feguenes" do } r_{n}\right)
$$

Nota-se que em 14.431 se fez una expanglo de laylor ate

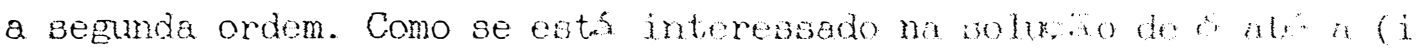
+ 1) iterexăo, então em 14.431 lem- se que

$$
\delta^{(i+1)}=\delta^{(i)}-H^{-1}\left(\delta^{(i)}\right) S\left(\delta^{(i)}\right)
$$

ou de forna equivalente

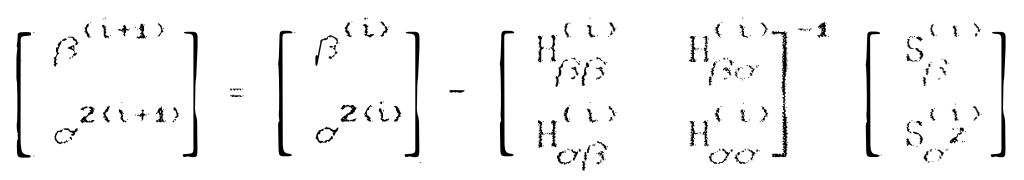

onde $\mathrm{H}^{-1}$ pode ser obtida a rartir dos resullados do llbNulinan \& SEARLF (1981), levando-se cm comideracado o befuinte finto:

$$
A=\left[\begin{array}{ll}
E & F \\
G & T
\end{array}\right]
$$

entào

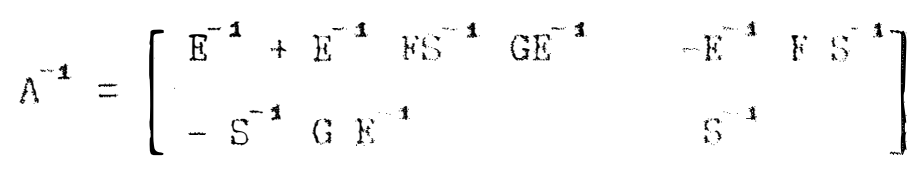

onde

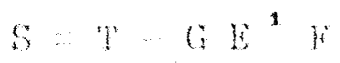

() resultado obtido en 14.451 former a solmot dos

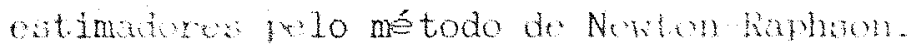

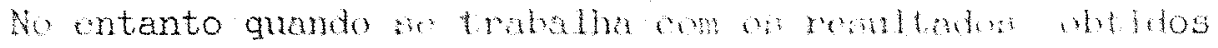




$$
\left[\begin{array}{l}
\beta^{(i+1)} \\
\sigma^{2(i+1)}
\end{array}\right]=\left[\begin{array}{cc}
\beta^{(i)}+I_{\beta \beta}^{-1(i)} & S_{\beta}^{(i)} \\
\sigma^{2(i)}+I_{\sigma \alpha}^{-1(i)} & \beta_{\alpha}^{(i)}
\end{array}\right]
$$

Resultado conhecido como "scores" de filihur. O critrio para se iniciar o procedimento é por exomplo estimar $\beta$ considerando que $\hat{V}=$ QMResí duo para o qual se unm os minimos quadrados ordinários, e prosseguir o procedimento al que.

i) $\quad|| \delta^{(i+1)}-\delta^{(i)}||<E$

ii) ||$\lambda\left(\delta^{(i+1)}\right)-\lambda\left(\delta^{(i)}\right)||<E$ com $\lambda=\sin (1$,

onde E é a precisão desejada.

Una vez obtidas as estimativas dos commmintei de: variância, elas podem ser usadas sem perda de rencralidube, na deterininasa dos BLUE e BLUP como na forma estudadn no cantulo 3. observação: Una condicão necessária para que tamto o a frorí tmo de Newton-Raphson como o "scoring" convirjan é que du mitriats H ... I em cada uma das iterasões, sejam não singulares e positivas nro definidas. Nestas condicões os dois procemon convermin rapidamente para as estimativas procuradas. 


\section{UM EXEMPLO ILUSTRATIVO}

Com o proposito de ilustrar alguns dos resultados desenvolvidos nas secóes anteriores, neste capí tulo apresenta-se un exemplo de um modelo misto com dois fatores sem interacäo

Seja o seguinte arranjo:

Fator 3

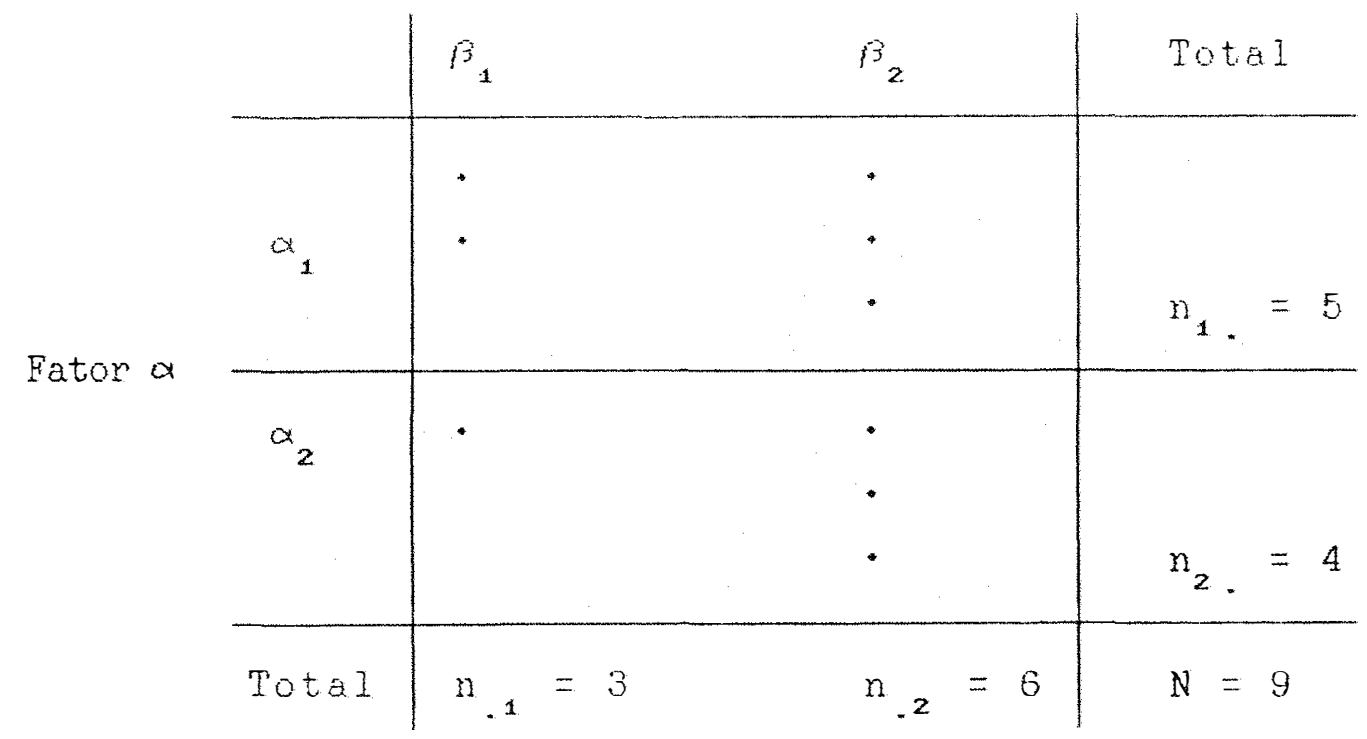

é claro que $N=\sum_{i=1}^{2} \sum_{j=1}^{2} n_{i j}=9$

- modelo que caracteriza o arranjo anterior sem levar en consideracão a nédia geral é 


$$
\begin{aligned}
& y_{i, j k}=\alpha_{i}+\beta_{j}+\epsilon_{i, k} \\
& i=1,2 ; j=1,2 ; k=1, \ldots, n_{1,}
\end{aligned}
$$

com a aleatório e $\beta$ fixo satiofazendo:

$$
\begin{aligned}
& \text { i) } E_{1}\left(\alpha_{2}\right)=0 ; E\left(\alpha_{1}^{2}\right)=\alpha_{\alpha}^{2} \text { i } \cdots 1, \\
& \text { i.j) } E\left(\varepsilon_{i, k}\right)=0 ; E\left(r_{i, j}^{2}\right)=y^{2} \\
& i=1,2 ; 1=1,2 ; k=1, \ldots n
\end{aligned}
$$

Observa-se então que o modelo 13.11 pode se eacrever mit fomi [3.1] fazendo

$$
\begin{aligned}
& { }_{1} \mathrm{Y}_{9}^{\prime}=\left[\mathrm{y}_{111} ; y_{112} ; y_{121} ; y_{122} ; y_{123} ; y_{213} ; y_{221}: y_{222} ; y_{223}\right] \\
& x_{0}=\left[\begin{array}{lllllllll}
1 & 1 & 0 & 0 & 0 & 1 & 0 & 0 & 0 \\
0 & 0 & 1 & 1 & 1 & 0 & 1 & 1 & 1
\end{array}\right] \\
& { }_{1}^{\beta}{ }_{2}^{\prime}=\left[\beta_{1} ; \beta_{2}\right] \\
& z_{0}^{*}=\left[\begin{array}{lllllllll}
1 & 1 & 1 & 1 & 1 & 0 & 0 & 0 & 0 \\
0 & 0 & 0 & 0 & 0 & 1 & 1 & 1 & 1
\end{array}\right] \\
& U_{2}^{*}=\left[a_{1}: x_{2}\right]
\end{aligned}
$$

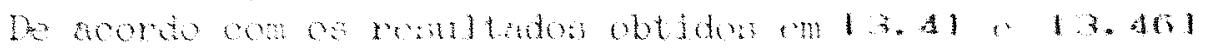
ten-se que 
63.

$\operatorname{Var}(y)=\sum_{t=0}^{1} Z_{i} Z_{i} \sigma_{i}^{2}=Z_{0} Z_{0} o_{0}^{2}+Z_{1} Z_{1} o_{1}^{2}=V$

[5. 2]

onde

$$
\begin{aligned}
& c_{0}^{2}=\sigma^{2} ; \alpha^{2}=\alpha_{c}^{2} \\
& Z_{0} Z_{0}=I_{a} \in Z_{1} Z_{1}={ }_{j=1}^{2} J_{n_{i}} .
\end{aligned}
$$

e por [3.41] a estrutura de $V$ obtida em [5.2] pode sor escrita na forma

$$
V=\sigma^{2} I_{\rho}+\sigma_{\alpha}^{2} \theta_{i=1}^{2} I_{i}
$$

con a qual, t, ainda considerando o resultado obtido em 13.481 . segue-se que

$$
V^{1}=\sum_{i=1}^{2} \frac{1}{\sigma^{2}\left(v^{2}+n_{i} \sigma_{\alpha}^{2}\right)}\left(c^{2} I_{n_{i}}+D\left(n_{i}, \sigma_{\alpha}^{2}\right)-\sigma_{\alpha}^{2} j_{n}\right) \quad 15.31
$$

fazendo-se en $[5.3]$

$$
\begin{aligned}
& k_{1}=\sigma^{2}\left(\sigma^{2}+n_{1} \alpha_{\alpha}^{2}\right)=\sigma^{2}\left(\sigma^{2}+5 \sigma_{\alpha}^{2}\right) \\
& k_{2}=\sigma^{2}\left(\sigma^{2}+n_{2} \alpha_{\alpha}^{2}\right)=\sigma^{2}\left(\sigma^{2}+4 \sigma_{\alpha}^{2}\right)
\end{aligned}
$$

entav 15.31 wie ser escrito om forma explícita com

$$
V^{-1}\left[\begin{array}{cc}
V_{1}^{-1} & 0 \\
0 & V_{2}^{-1}
\end{array}\right]
$$

onstis 
64.

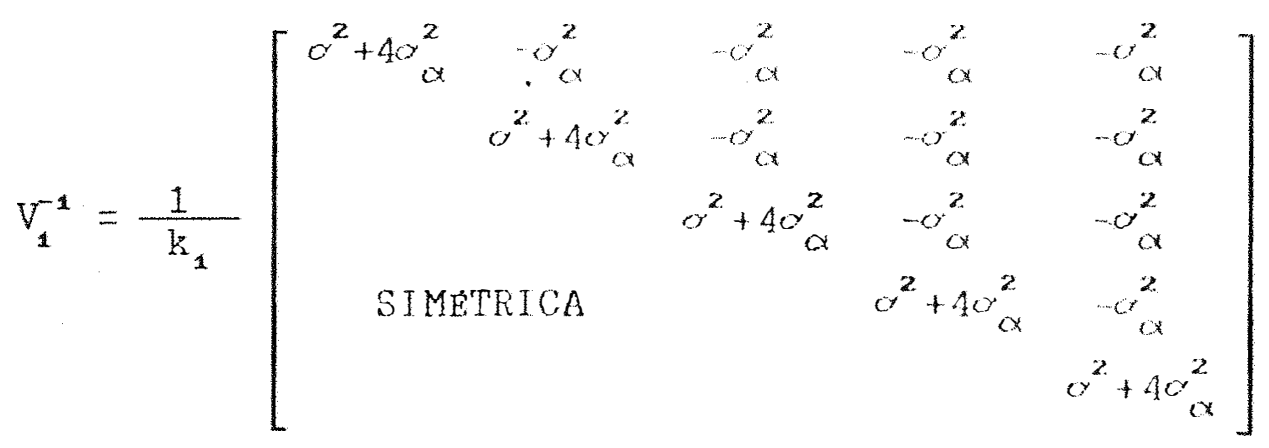

e

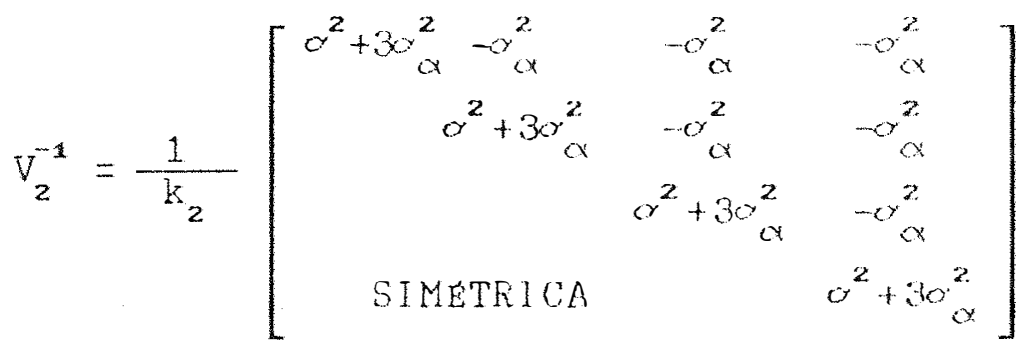

Cono o intearesse inicial nente trabalho foi a crimaca no modelo [3.1] de $\beta$ e.H : então agora no contexto do modelo lis.1] tem-se de [3.11] que

$$
\beta^{0}=\left(X V^{-1} X\right)^{-1} X V^{-1} y
$$

neste caso

$$
\begin{aligned}
& X^{\prime} V^{-1} X=\frac{1}{k_{1} k_{2}}\left[\begin{array}{cc}
\left(o^{2}+3 \alpha_{\alpha}^{2}\right)\left(2 k_{2}+k_{1}\right) & -3 \alpha_{(2)}^{2}\left(2 k_{2}+k_{1}\right) \\
-3 \alpha_{\alpha}^{2}\left(2 k_{2}+k_{1}\right) & \left.3 k_{2}+k_{1}\right)+3 \alpha_{\alpha}^{2}\left(2 k_{2}+k_{1}\right)
\end{array}\right]
\end{aligned}
$$

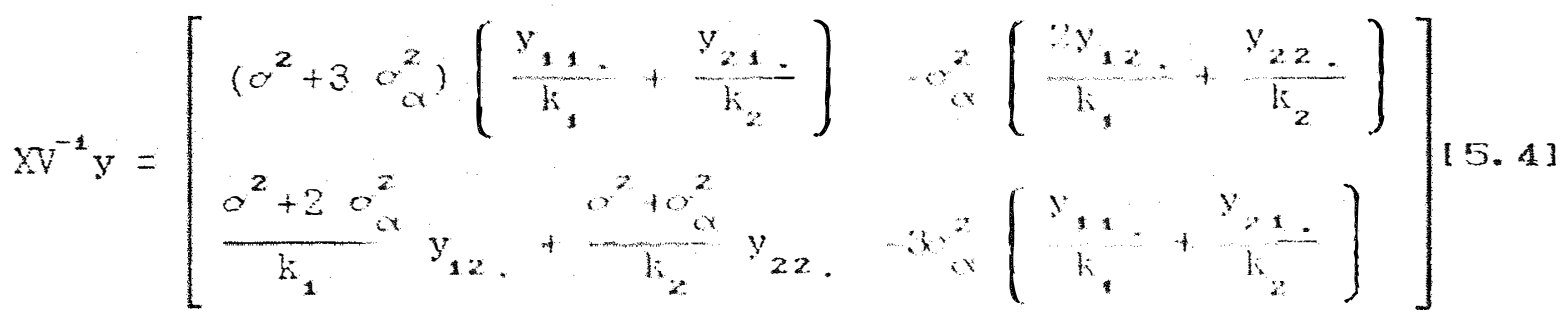




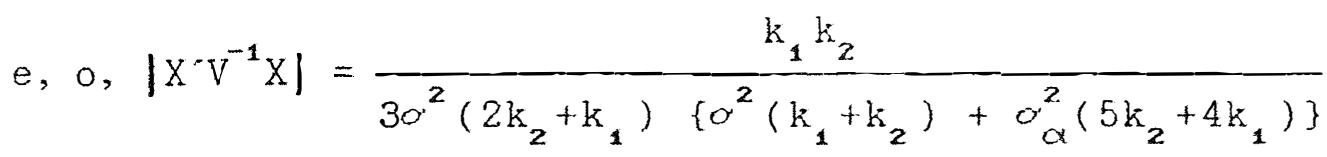

(onde $\mid$ | é o determinante)

Deste modo

$\left(X^{-1} V^{-1} X\right)^{-1}=\frac{1}{\left|X V^{-1} X\right|}\left[\begin{array}{cc}3 \sigma^{2}\left(k_{2}+k_{1}\right)+3 \sigma_{\alpha}^{2}\left(2 k_{2}+k_{1}\right) & 3 \sigma_{\alpha}^{2}\left(2 k_{2}+k_{1}\right) \\ 3 \gamma_{\alpha}^{2}\left(2 k_{2}+k_{1}\right) & \left(\sigma^{2}+3 \sigma_{\alpha}^{2}\right)\left(2 k_{2}+k_{1}\right)\end{array}\right]$ [5.5]

Do produto das matrizes $[5.5]$ e $[5.4]$ obtem-se a estimativa para $\beta$, ou seja,

$$
\left.\hat{\beta}^{-}=\hat{\beta}_{i} ; \hat{\beta}_{2}\right]
$$

Para se obter a estimativa de U, na estrutura do modelo $[3.1]$, tem-se de [3.18] que

$$
K V^{-1}={\underset{i=1}{2}}_{i=1} D\left(\frac{\alpha_{\alpha}^{2}}{\sigma^{2}+n_{i} \cdot \sigma_{a}^{2}}\right)
$$

e entäo

$$
\hat{\mu}=\left[\begin{array}{c}
\frac{\sigma_{\alpha}^{2}}{\sigma^{2}+4 \alpha_{\alpha}^{2}}\left(y . .-x \beta^{o}\right) \\
\frac{\sigma_{\alpha}^{2}}{\sigma^{2}+4 \sigma_{\alpha}^{2}}\left(y, 2,-X \beta^{0}\right)
\end{array}\right]
$$


E claro,neste desenvolvimento, que tanto $f$ rom is dependem linearmente dos componentes aleatórios das varincias $\alpha^{2}$ e $\alpha_{\alpha}^{2}$, dai a necessidade de se obter a estinacão delau. Assim, pela decomposisão ortogonal

$$
\begin{aligned}
& y_{i j k}=\left[\frac{1}{n_{i}} y_{i} \ldots \frac{1}{n_{i j}} y_{i j}\right]+\left[\frac{1}{n_{1} \ldots}-\frac{1}{n_{i j}} y_{i j}\right]+ \\
& +\left[y_{2 j k}-\frac{1}{n_{i}} y_{i} \ldots-\frac{1}{n_{, j}} y_{j j}+\frac{1}{n_{i j}}\right]+\frac{1}{n_{1} y_{1 .}}
\end{aligned}
$$

onde $n_{i}=\sum_{j=1}^{2} n_{i j} ; n_{-j}=\sum_{i}^{2} n_{i j}$

$$
\text { e } \quad N=\sum_{i=1}^{2} \sum_{j=1}^{2} n_{i j}
$$

dos resultados obtidos em $[3.39],[3.40],[3.4: 1,1,1.41,[4.15]$ [4.16] para dados não balanceados,

$$
V=\lambda_{0} A_{0}+\lambda_{1} A_{1}
$$

onde para se obter as matrizes $A_{0}$ e $A_{1}$, levando cm consideracão a decomposicão ortogonal:

$$
\frac{1}{n_{i}} v_{i} \Rightarrow\left[\begin{array}{cccc}
\frac{1}{5} & J_{5} & 0 \\
& 0 & \frac{1}{4} & J_{4}
\end{array}\right]=k
$$




$$
\frac{1}{n_{i j}} y_{i j .} \Rightarrow\left[\begin{array}{ccccc}
-\frac{1}{2} & J_{2} & 0 & 0 & 0 \\
0 & -\frac{1}{3} J_{3} & 0 & 0 \\
0 & 0 & J_{1} & 0 \\
0 & 0 & 0 & \frac{1}{3} J_{3}
\end{array}\right]=G
$$

ou na sua forma explí cita

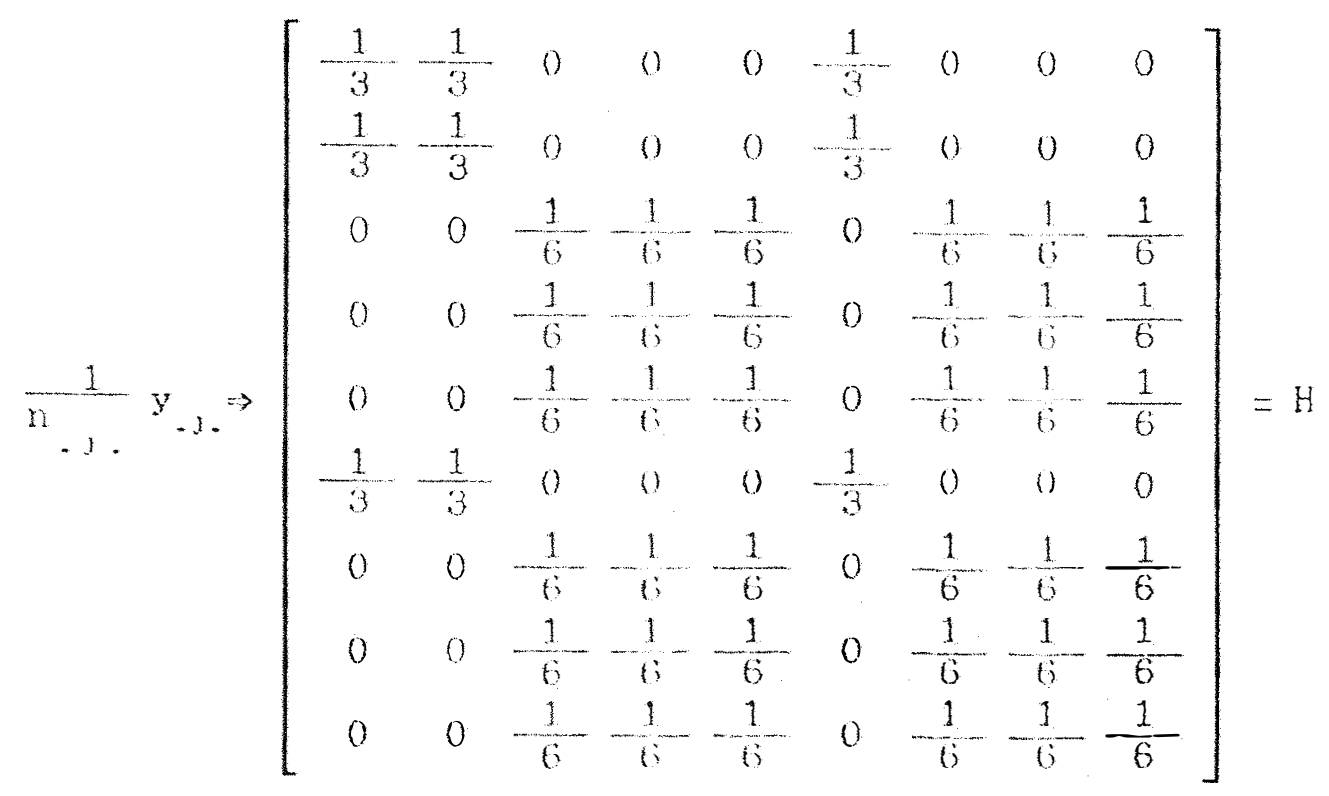

de tal modo que

$$
\begin{aligned}
& A_{0}=\left[I_{n}-F-H+G\right] \\
& A_{1}=\underbrace{2}_{1} \frac{1}{n_{1}} n_{1}-\frac{1}{9} n_{0}
\end{aligned}
$$

ou na sua forma explí cita 
68.

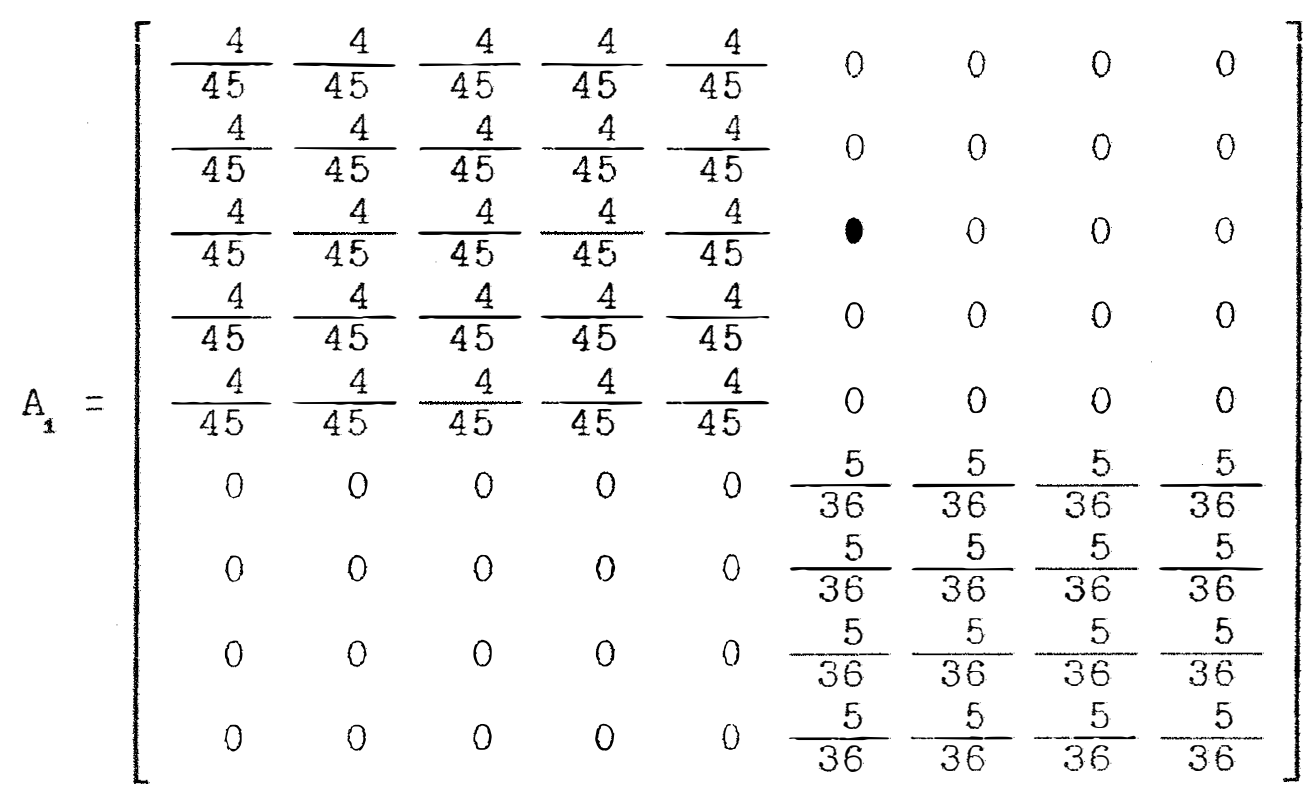

$E$ des resultados obtidos em $[4.22]$ e $[4.31]$ segre-se que:

$$
\hat{\lambda}_{0}=\frac{y^{\prime} A_{0} y}{\operatorname{ran}\left(A_{0}\right)}
$$

onde

$$
\begin{aligned}
& y^{\prime} A_{o} y=\sum_{i=1}^{2} \sum_{j=1}^{z} \sum_{k=1}^{n_{i} j} y_{i j k}^{2}+\sum_{i} \sum_{j} \frac{1}{n_{i j}} y_{i j .}^{2}- \\
& -\left(\frac{1}{3} y_{.1}^{2}+\frac{1}{6} y_{.2}^{2}\right)-\left(\frac{1}{5} y_{1}^{z}+\frac{1}{4} y_{z}^{2}\right)
\end{aligned}
$$

e $\operatorname{ran}\left(A_{0}\right)$,é o rank da matriz $A_{0}$ ou tankém $\operatorname{ran}\left(A_{0}\right)=N-S$, con $S:$ número de caselas não vazias.

Desse modo, e pelo fato do resultado obtido en $[4.30]$ onde

$$
E\left(y^{\prime} A_{0} y\right)=(N-S) o^{2}
$$


60.

segre-se que

$$
\begin{aligned}
& \hat{\lambda}_{0}=\hat{o}^{2}=\frac{1}{5}\left[\sum_{i}^{2} \sum_{j}^{2} \sum_{k}^{n_{i j}} y_{i j k}^{2}+\sum_{i}^{2} \sum_{j}^{2} \frac{1}{n_{i j}} y_{i j}^{2}-\right. \\
& -\left[\frac{1}{3} y^{2}+\frac{1}{6} y^{2} y_{-2}\right]-\left[\frac{1}{5} y_{1}^{2}+\frac{1}{4} y_{z}^{2} \ldots\right]
\end{aligned}
$$

de forma semelhante

$$
\begin{aligned}
y^{\prime} A_{1} y & =\frac{n_{2}}{N n_{1}} y_{1}^{2}+\frac{n_{1}}{N n_{2}} y_{2} \ldots \\
& =\frac{4}{45} y_{1}^{2}+\frac{5}{36} y_{2}^{2} \ldots
\end{aligned}
$$

e por [4.31]

$$
E\left(y-A_{1} y\right)=\left(N-k_{1}\right) \sigma_{\alpha}^{2}+\sigma^{2}
$$

$\operatorname{com} k_{1}=\frac{1}{N} \sum_{i} n_{i}^{2}=\frac{41}{9}$

e assin

$$
E\left(y^{\prime} A_{1} y\right)=\frac{40}{9} c_{\alpha}^{2}+\alpha^{2}
$$

e por $[4.35]$ segue-se que

$$
\hat{\sigma}_{0}^{2}=\frac{9}{40}\left[\hat{\lambda}_{1}-\hat{\lambda}_{0}\right]
$$

onde 


$$
\hat{\lambda}_{1}=\frac{y^{\prime} A_{1} y}{r\left(A_{1}\right)}=\frac{1}{2}\left[\frac{4}{45} y_{1}^{2}+\frac{5}{36} y_{2}^{2}\right]
$$

desse modo

$$
\begin{aligned}
\hat{\sigma}_{\alpha}^{2} & =\frac{9}{40}\left\{\frac{1}{2}\left[\frac{4}{45} y_{1}^{2}+\frac{5}{36} y_{2}^{2} \ldots\right]-\right. \\
& -\frac{1}{5}\left[\sum_{i}^{2} \sum_{j}^{2} \sum_{i}^{n_{i j}} y_{i j k}^{2}+\sum \sum \frac{1}{n_{i j} y_{i j .}^{2}}\right] \\
& \left.\left.-\left[\frac{1}{5} y_{1}^{2}+\frac{1}{4} y_{z}^{2}\right]-\left[\frac{1}{3} y_{.1 .}^{2}+\frac{1}{6} y_{.2}^{2}\right]\right]\right\}
\end{aligned}
$$

Uma vez obtidas as estinativas dos componentes, pode-se obter combinacões lineares cono em [3.33]; essas combinasóes dependem da natureza do problema e do interesse do pesquisador. 


\section{CONCLUSÖES}

Nos modelos de efeitos mistos, o uso dos minimos quadrados pondexados facilitou a estimara dos preditores e das funcões lineares associadas aos efeitos aleatórios e fixos do modelo.

0 uso da funsão de Lagrange, na equarão [3.23] sujieita às condisões [3.22] facilitou a caracterizasão dos preditores os quais como observa-se em [3.32], dependem da forma linear da estimasão dos efeitos fixos e aleatórios do modelo.

O método apresentado para estruturar a matriz dos componentes de variancia, assim como o cálculo da sua inversa, fornece uma ferramenta simples na obtencão dos preditores Iineares.

Observou-se que quando a estrutura dos dados for balanceada, e a matriz associada aos efeitos fixis for de posto coluna completo, então o BLUE não vai depender do componente aleatório do modelo.

Ao estabelecer uma relasão entre os sub-índices do modelo e as particões ortogonais em termos dos produtos de Kronecker de matrizes identidades de orden $s$ (Is) quadradas, de elenentos unitários de orden $=\left(T_{s}\right)$, simplificou-se a construsä̃o das formas quadráticas, e com isto, a construrão das funsões de 
verossimilhanca para se obter, desse modo, a estimacão dos componentes de variância. 


\section{REFERENCLI AS EIBLIOGRAFICAS}

ANDERSON, R. L. \& EANCROFT, T. A. Statistical theory in research, MoGran Hill, New York, 1952. 420 pp.

CORBELL, R. R. \& SEARLE, S. R. Restrioted maximun likelihood (REML) estimation of variance components in the mixed model. Technometrics. 1 vol. 18, n० 1, 435-440. 1976.

DODCE, I. Analysis of Experiments with Missing Data. John Wiley, New York. 1985.

GOLDSTEIN, H. Multilives mixed linear model analysis wsine iterative generalized least squares. Biometrika. 49: 350-365. 1986.

GOODNIGHT, J.H. \& SPEED, F. M. Computing expected mean squares. Biometrics, 36: 123-125. 1980.

HARTLEY, H. O. Expectation, Variances and cavariances of ANOVA mean square by syntesis Biometrics. 23: 103-114. 1967. 
HARTLEY, H. O. \& RAO, J. N. K. Maximum likelihood estimation for the mixed analyses of variance nodel. Biometrika. 52: 93-103. 1967.

HARTLEY, H. O. \& VAUGHN, W. K. A. A computer program for the nixed analysis of variance nodel based on maximum likelihoni. Ames of Iowa. pp. 129-144. 1972.

HARVEY, $W$. R. Conputing procedures for a benerulized least-aquares analysis. Program colorado state univelsity. FP. 50. 1964.

HARVEY, W. R. Estination of variance and covariances cumpunenti: in the mixed models. Biometrics. 1970. 32: 445-4(6).

HARVEY, W. R. Least aquares analysis of data. United itiatc:; departament of agricultural; Agricultural research. (1) $\mathrm{m}$. 1979.

HEMMERLE, W. J. \& HARTLEY, H. O. Computing maximum likuthood estimate for the mixed A.O.V. Model using the $W$. Transformation. Technometrics. 4:15, n० 4, 819-8331. 19\%7,

HENDERSON, C, R. Estimation of variance and covinmm... components. Biometrics. 49: 226-257. 1953. 
HENDERSON, C. R. Simulation to examine distributions of estimators of variances and ratios of variances. J. Dairy Sci. $61: 267-273 . \quad 1977$.

HENDERSON,C. R. J.r. Analysis of covariance in the nixed model high-level mon-honogeneaus, and randon repressions. Bi ometrics, 38: 623-649. 1982 .

HENDERSON, C. R. Applications of linear models in animal breading. University of guelph. 1984a.

HENDERSON, C. R. Estimation of variances and covariances under. nultiple trait models. J. Dairy Sei, 67: 1581-1589. 1984b.

HENDERSON, H. V. \& SEARLE, S. R. Veo and Vech operators for matrices, with sone uses in jacobians and mutivariate Statistics. Can. J. Stat., 7: 65-81. 1979.

HENDERSON, H. V. \& SEARLE, S. R. On deriving the inverse of a some of matrices. Siam Revew, 53-60. 1981.

HENDERSON, C. R.; SEARLE, S. R. \& SCHOEEEER, L. R. The invariance an calculation of method 2 for estinating variance comprents. Eiometrics, 30: 583-588. 1974.

HOCKING, R. R. The analysis of 1 inear models. Brooks/cole fuhl lishine company. 1985. 


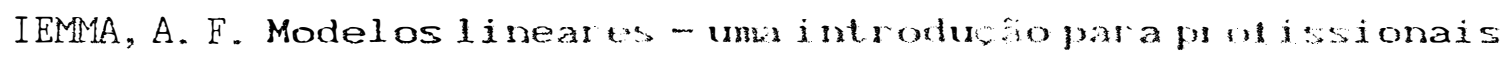

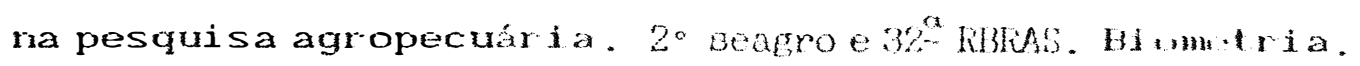
1987.

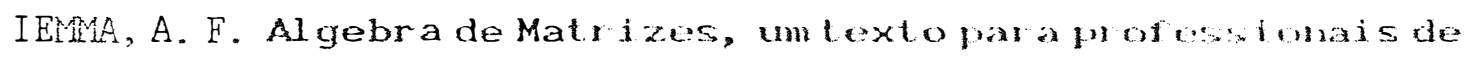
ciências aplicadas ESAlR/USE. 1985.

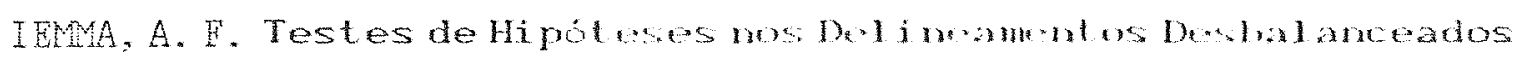
F.S.A. Gembloux Belgique. 1991.

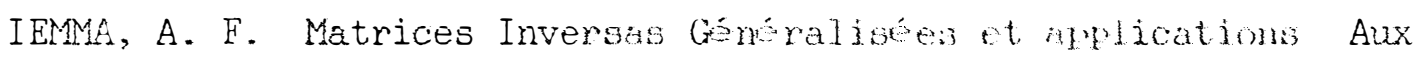
Modéles Linéares. Notes stat Inform. Gembloux Belgique. 1992.

JENRICH, R. I. \& SCHLUCHTH, M. D. Umbalamed repetead modures models with struetured covariance matriso;. Hiometri,s, 42: 805-820. 1986.

LA MOTTE, L. R. Quadratic estimation of variance comments. Biometrics. 29: 311-330. 1973.

MENDEZ. I. Estudio de los nodelos mixtos. Commi aminmes Menicas UNAM, MÉxico. 1978.

PATTERSON, H. D. \& THMMSON, h. hinvery of introlock

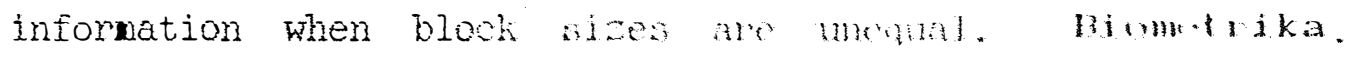
36: $538-545 . \quad 1971$. 
RAO, C. R. Estimation of variance and covariance components. Journal of Multivariate Analysis. 42: 257-275. 1971.

RAO, C. R. Estimation of variance and covariance components in linear models. JASA, 69: 112-115. 1972.

SCHEFFE, H. The analysis of variance, John Wiley, New York. 1959.

SEARLE, S. R. Li near Models, John Wiley, New York.1971.

SEARLE, 5. R. Matrix Algebra Useful for Statistics, John Wiley, New York. 1982.

SEARLE, S. R. Li near models for unbal anced data, John hiley, New York. 1987.

SEARLE, S. R. Mixed models and unbalanced deta. Were from where at and where to? Commum. Statist. Theory Meth, 17(4): 935-968. 1980

SEARLE, S. R. \& HENDERSON, H. V. Dispersion Matrizes for variance components mociels. JASA, 34: 465-470. 1979.

SPEED, F. M. \& HOCKING, R. R. Compution of expectations, variances and covariances of Anova mean square. Biometrics. 30: $157-160 . \quad 1974$ 
SAS user's Guide: Statistics, 1982. Ediction, SAS Institute Inc. Box 800, Capry, North Carolina, 27511. 


\section{APENDICB}

\section{PROGRAMA EM MATRIX/IML DO SAS}

/* No seguinte programa exemplifica-se a partir do modelo

[3.43] e a extenção é imediata.*/

\section{STATRT DISE;}

002 RESET FW=5;

$003 \mathrm{NC}=\mathrm{NCOL}(\mathrm{X} 1)$; /* numero de colunas da parte fixa do modelo*/

$004 \mathrm{NF}=$ NROW(X1); /* numero de linhas da parte fixa do modelo*/

$005 \mathrm{~K}=\{\mathrm{n}\} ; / *$ numero de observaçธ̃es por casela*/

$006 \mathrm{~N}=\mathrm{NF} * \mathrm{NC} * \mathrm{~K}$; /* Total de unidades experimentais $* /$

$007 \mathrm{IN}=\mathrm{I}(\mathrm{N})$;

$008 \mathrm{JN}=\mathrm{J}(\mathrm{N})$;

$009 \mathrm{II}=\mathrm{I}(\mathrm{NC})$;

$010 \mathrm{IJ}=\mathrm{I}(\mathrm{NF}) ; \quad / *$ Nas instruç Ces 7-14 obtem-se as matrizes

$011 \mathrm{IK}=\mathrm{I}(\mathrm{K})$; identicas e quadradas de orden um*/

$012 \mathrm{JI}=\mathrm{J}(\mathbb{N C})$;

$013 \mathrm{JJ}=\mathrm{J}(\mathrm{NP})$;

$014 \mathrm{JK}=\mathrm{J}(\mathrm{K})$;

015 IIJ=II@IJ@JK;

016 IJI=II@JJ@IK;

017 JII=JI@IJ@IK; 
018 IJJ=II@JJ@JK;

019 JIJ=JI@IJ@JK;

020 JJI=JI@JJ@IK;

$021 \mathrm{AM}=\mathrm{JN} / \mathrm{N}$ :

$022 \mathrm{AN}=\mathrm{IJJ} /(\mathrm{NF} * \mathrm{~K})$;

$023 \mathrm{AP}=\mathrm{JIJ} /(\mathrm{NC} * \mathrm{~K})$;

$024 \mathrm{AQ}=\mathrm{IIJ} / \mathrm{K}$;

025 FREE=II IJ IK JI JJ JK;

$026 / *$ DE1=IN || IIJ || IIJ || JII;

027 FREE IJI JII;

028 DE2=IJJ || JIJ;

$029 \mathrm{DE} 3=\mathrm{JJI}|| \mathrm{JN}$;

030 FREE JJI JN;

031 DELTA= DE1 || DE2 || DE3; /*Com este resultado obtem-se a equaçå $3.39 * /$

032 FREE DE1 DE2 DE3 ;

$033 \mathrm{AO}=\mathrm{IN}-\mathrm{AQ}$;

$034 \mathrm{A1}=\mathrm{AN}-\mathrm{AM}$;

$035 \mathrm{~A} 2=\mathrm{AP}-\mathrm{AM}$;

$036 \mathrm{~A} 3=\mathrm{AQ}-\mathrm{AN}-\mathrm{AP}+\mathrm{AM}$;

/*Partiçธes ortogonais apresentadas na equação $4.15 * /$

$037 / * A 3 \mathrm{P}=\mathrm{AQ}-\mathrm{AN}-\mathrm{A} 2 ; \quad * /$

$038 \mathrm{TAO}=\mathrm{ROOT}(\mathrm{AO})$;

039 TA1=ROOT(A1);

040 TA2=ROOT (A2);

/*Calcula os traços das equaçర్s 4.20 e 4.21*/

$041 \mathrm{TA3}=\mathrm{ROOT}(\mathrm{A3})$;

$042 / *$ A11=A1-TA1*TA1; */

$043 \mathrm{DAO}=\mathrm{TAO}[\operatorname{LOC}(\operatorname{VBCDIAG}(\mathrm{TAO})>0)$, ];

044 FREE TAO;

045 DA1=TA1[LOC $(\operatorname{VBCDIAG}(\mathrm{TA1})>0)$, ];

046 FREE TA1;

047 DA2=TA2[LOC $(\operatorname{VECDIAG}(\mathrm{TA2})>0)$, ];

048 FREE TA2;

049 DA3=TA3[LOC $(\operatorname{VECDIAG}(\mathrm{TA} 3)>0)$, ];

050 FREE TA3;

051 RAO= NROW (DAO); 
052 RA1 = NROW (DA1);

/* Calcula os postos das matrizes

053 RA2 = NROW(DA2); apresentadas na equaçăo 4.16*/

054 RA3 = NROW(DA3);

055 PREB DAO DA1 DA2 DA3;

$056 \mathrm{TI}= \begin{cases}10,10 \\ 0\end{cases}$

/* Neste caso supre-se que $n=2 * /$

$057 / * T I(2,2)=\mathrm{NC} ; * /$

058 TI $[\{2\},\{2\}]=N C$;

$059 \mathrm{TJ}=\left\{\begin{array}{lll}0,1 & 1\end{array}\right\}$;

$060 \mathrm{TJ}[\{2\},\{2\}]=\mathrm{NP}$;

$061 / * \mathrm{~T} J(2,2)=\mathrm{NP} ; 8 /$

$062 \mathrm{TK}=\{10,10\}$;

$063 \operatorname{TK}\{\{2\},\{2\}]=K$;

$064 \mathrm{TP}=\mathrm{TI} \mathrm{TJ} @ \mathrm{TK}$;

/*Calcula a matriz Tp */

$065 \mathrm{ITP}=\mathrm{INV}(\mathrm{TP})$;

/*Calcula a inversa de $\mathrm{T}_{\mathrm{p}} * /$

066 PRINT TP ITP;

067 PRBB TI TJ TK;

$068 / * \quad \mathrm{BAO}=\operatorname{BIGVAL}(\mathrm{AO})$;

$069 \mathrm{BA1}=\operatorname{BIGVAL}(\mathrm{A} 1)$;

070 BA2 = $\operatorname{BIGVAL}(\mathrm{A2})$;

071 BA3= $\operatorname{EIGVAL}(\mathrm{A3})$;

072 CALL BIGEN(BO,MO,AO);

073 CALL BIGEN(B1,M1,A1);

074 CALL BIGEN(B2,M2,A2);

075 CALL BIGEN( $B 3, M 3, A 3) ; * /$

$076 \mathrm{LO}=\left(\mathrm{Y}^{\circ} * \mathrm{AO} * \mathrm{Y}\right) / \mathrm{RAO}$;

$077 \mathrm{~L} 1=\left(\mathrm{Y}^{*} * \mathrm{~A} 1 * \mathrm{Y}\right) / \mathrm{RA} 1$;

$078 \mathrm{~L} 2=\left(Y^{\circ} * \mathrm{~A} 2 * \mathrm{Y}\right) / \mathrm{RA} 2$;

/*Resultados para obter-se as estimativas da equação $4.22 *$ /

$079 \mathrm{~L} 3=\left(\mathrm{Y}^{\circ} * \mathrm{~A} 3 * \mathrm{Y}\right) / \mathrm{RA3}$;

080 PRINT LO,L1,L2,L3;

081 EREB A0,A1,A2;

$082 \mathrm{SCB}=\mathrm{LO}$;

$083 \mathrm{SCR}=(\mathrm{L} 3-\mathrm{LO}) / \mathrm{K}$;

$084 \mathrm{SC} 1=(\mathrm{L} 1-\mathrm{L} 3) /(\mathrm{NF} * \mathrm{~K})$;

/*Obtem-se as estimativas de componentes da variancia*/

$085 \mathrm{SC2}=(\mathrm{L} 2-\mathrm{L} 3) /(\mathrm{NC} K \mathrm{~K})$;

086 PRINT SCE,SCR,SC1.SC2;

Q87 FREB LO L1 L2 L3; 
088 IF SCR $[\{1\},\{1\}]<0$ THEN SCR $=\{0\}$;

089 IF SC1 $\{1\},\{1\}]<0$ THEN SC1 $=\{0\}$;

090 IF $\operatorname{SC} 2[\{1\},\{1\}]<0$ THEN SC2 $=\{0\}$;

$091 \mathrm{CER}=\{0\}$;

$092 \mathrm{TETA}=\mathrm{SCE} / / \mathrm{SCR} / / \mathrm{CER} / / \mathrm{CER} / / \mathrm{CER} / / \mathrm{SC} 2 / / \mathrm{SC} 1 / / \mathrm{CER}$;

093 PRINT TETA;

$094 / * \mathrm{TET} * \mathrm{CER} / / \mathrm{CER} / / \mathrm{SC} 2 / / \mathrm{CER} / / \mathrm{SC} 1 / / \mathrm{CER} / / \mathrm{SCR} / / \mathrm{SCE} ; * /$

$095 / *$ TETA P=TETA; $* /$

$096 / * \mathrm{~V}=$ DELTA $*$ TETA; $* / / *$ Resultado da equação $3.41 * /$

097 /* DELTA $=$ IN || IIJ || IJI || JII || IJJ || JIJ || JJI || JN;

099 PRINT DELTA; */

$100 \mathrm{~V}=$ SCE\#IN + SCR\#IIJ + SC2\#JIJ + SC1\#IJJ;

101 PRINT V;

102 /*RREB DELTA; */

103 PREE IIJ IJJ JIJ;

104 PREE IJI JII JJI JN;

$105 \mathrm{IV}=\operatorname{INV}(\mathrm{V})$;

/*alcula a matriz da equaçăo $3.42 * /$

106 PRINT IV ;

107 LP=TP*TETA; /*Combinaçธes lineares da equaçฐ̃o $3.44^{\circ} * /$

108 PREE TP TETA;

109 ILP=1/(LP);

110 IVV= ITP*ILP;

111 PRINT IVW, LP, ILP;

112 FREE LP, ILP, ITP;

113 * IV=ITP*ILP*DELTA $; * /$

$114 \mathrm{R}=\mathrm{SCE} \# \mathrm{IN}$;

$115 \mathrm{D} 1=\mathrm{SC} 1 \# \mathrm{I}(\mathrm{NC})$;

$116 \mathrm{D} 2=\mathrm{SC} 2 \# \mathrm{I}(\mathrm{NP})$;

117 D3=SCR\#I (NF\#NC);

$118 \mathrm{D}=\mathrm{BLOCK}(\mathrm{D} 1, \mathrm{D} 2, \mathrm{D} 3)$;

119 FREE D1D2D3;

$120 \mathrm{Z} 1=\left\{\begin{array}{llllllllllllllllll}1 & 0 & 1 & 0,1 & 0 & 1 & 0,1 & 0 & 0 & 1,100 & 0 & 1,0110,\end{array}\right.$

$\begin{array}{lllllllllll}0 & 1 & 1 & 0,0 & 1 & 0 & 1,0 & 1 & 0 & 1\} ;\end{array}$

$121 \mathrm{Z2}=\mathrm{Z} 1[, 1] \# \mathrm{Z} 1[, 3]$;

$122 \mathrm{Z3}=\mathrm{Z} 1[, 1] \# \mathrm{Z} 1[, 4]$; 
$123 \mathrm{Z} 4=\mathrm{Z} 1[, 2] \sharp \mathrm{Z} 1[, 3]$;

$124 \mathrm{Z} 5=\mathrm{Z} 1[, 2] \# \mathrm{Z} 1[, 4]$;

$125 \mathrm{Z}=\mathrm{Z1}|||\mathrm{Z2}||\mathrm{Z3}||\mathrm{Z4}||| \mathrm{Z5}$;

126 FREB Z1 Z2 Z3 Z4 Z5 ;

$127 \mathrm{KZD}=\mathrm{Z} * \mathrm{D}$;

128 PRINT R Z D KZD;

$129 \mathrm{~B} 0=\operatorname{INF}\left(\mathrm{X}^{\circ} * \mathrm{IV} * \mathrm{X}\right) * \mathrm{X}^{*} * \mathrm{IV} * \mathrm{Y} ; / *$ Bstimaçăo de $\beta$ na equaç $3.11 * /$

130 PRINT BO;

$131 \mathrm{U}=\mathrm{KZD} \mathrm{D}^{*} * \mathrm{~V}^{*} *(\mathrm{Y}-\mathrm{X} \# \mathrm{BO})$;

132 FINISH;

133 RUN DISE; 